\title{
Visual Stimulus Content in V4 Is Conveyed by Gamma-Rhythmic Information Packages
}

\author{
Dmitriy Lisitsyn, ${ }^{1}{ }^{\circledR}$ Iris Grothe, ${ }^{2,3}$ Andreas K. Kreiter, ${ }^{3}$ and Udo A. Ernst ${ }^{4}$ \\ ${ }^{1}$ Computational Neurophysics Lab, Institute for Theoretical Physics, University of Bremen, Bremen 28359, Germany, ${ }^{2}$ Ernst Strüngmann Institute \\ (ESI) for Neuroscience in Cooperation with Max Planck Society, Frankfurt 60528, Germany, ${ }^{3}$ Center for Cognitive Science, Brain Research Institute, \\ University of Bremen, Bremen 28359, Germany, and ${ }^{4}$ Computational Neurophysics Lab, Institute for Theoretical Physics, University of Bremen, \\ Bremen 28359, Germany
}

Selective visual attention allows the brain to focus on behaviorally relevant information while ignoring irrelevant signals. As a possible mechanism, routing-by-synchronization was proposed: neural populations receiving attended signals align their gamma-rhythmic activity to that of the sending populations, such that incoming spikes arrive at excitability peaks of receiving populations, enhancing signal transfer. Conversely, non-attended signals arrive unaligned to the receiver's oscillation, reducing signal transfer. Therefore, visual signals should be transferred through gamma-rhythmic bursts of information, resulting in a modulation of the stimulus content within the receiving population's activity by its gamma phase and amplitude. To test this prediction, we quantified gamma-phase-dependent stimulus content within neural activity from area V4 of two male macaques performing a visual attention task. For the attended stimulus, we find highest stimulus information content near excitability peaks, an effect that increases with oscillation amplitude, establishing a functional link between selective processing and gamma-activity.

Key words: attention; gamma; information routing; selective processing; V4; visual system

Significance Statement

The ability to focus on the behaviorally relevant signals is essential for the brain to cope with the continuous, high-dimensional stream of sensory information it receives. What are the neural mechanisms which allow such selective processing in the visual system? We analyzed data from area $\mathrm{V} 4$ and found that the amount of visual signal information content is tightly linked to the phase of local gamma-rhythmic activity, with maximal signal content occurring near peaks of neural excitability. Our investigations provide direct evidence that selective attention relies on rhythmic temporal coordination between visual areas, and establish novel methods for pinpointing pulsed transmission schemes in neural data.

\section{Introduction}

Visual information processing is computationally demanding, requiring the brain to handle a continuous, high-dimensional stream of sensory input signals. Selective attention helps to reduce this computational complexity by focusing on signals which are

Received Mar. 21, 2020; revised Sep. 5, 2020; accepted 0ct. 15, 2020.

Author contributions: D.L. and U.A.E. designed research; D.L., I.G., A.K.K., and U.A.E. performed research; D.L. analyzed data; D.L. and U.A.E. wrote the paper.

This work was supported by The Federal Ministry of Education and Research (BMBF) Bernstein Group for Computational Neuroscience Bremen Grant 01G00705, Innovationswettbewerb Medizintechnik Grant 01 EZ 0867, and Bernstein Award Udo Ernst Grant 01GQ1106; the Deutsche Forschungsgemeinschaft (DFG) Priority Program 1665 Grants ER 324/3 and KR 1844/2-2; the University of Bremen's Research-Focus Neurotechnology, Creative Unit I-See, and Zentrum für Kognitionswissenschaften; and the Leibniz Graduate School for Primate Neurobiology (I.G.). We thank K. Thoß, R. Hakizimana, and K. Taylor for monkey care and training and D. Rotermund for support in data preprocessing.

The authors declare no competing financial interests.

Correspondence should be addressed to Dmitriy Lisitsyn at dmitriy@neuro.uni-bremen.de.

https://doi.org/10.1523/JNEUROSCI.0689-20.2020

Copyright $(2020$ the authors behaviorally relevant at the expense of other, irrelevant signals (Lavie, 1995).

Selective processing is already observed at the single neuron level: when presented with two stimuli inside their receptive fields (RFs) of which one is attended, V4 neurons respond primarily as if only this stimulus was present (Moran and Desimone, 1985; Reynolds et al., 1999; Grothe et al., 2018). However, there is only a small attention-dependent modulation of firing rates of the $\mathrm{V} 1 / \mathrm{V} 2$ populations providing the input signals from the two visual stimuli to V4 (Moran and Desimone, 1985; Motter, 1993; Luck et al., 1997; McAdams and Maunsell, 1999; Mehta et al., 2000; Salinas and Sejnowski, 2000). Hence, selective responses in downstream areas cannot result exclusively from upstream rate modulations, suggesting that selective attention relies on a different mechanism to dynamically change effective connectivity depending on task demands.

One influential idea proposes interareal synchronization of oscillatory neural activity in the gamma-band $(40-100 \mathrm{~Hz})$ to enact dynamic modulation of effective connectivity between 
A

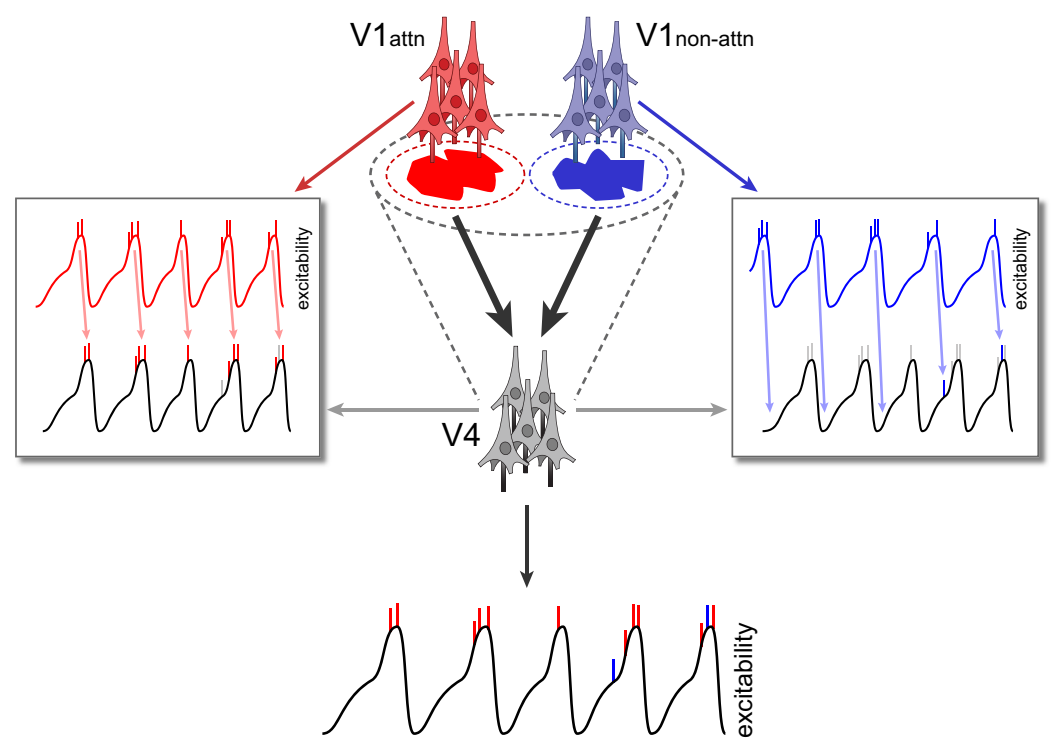

B
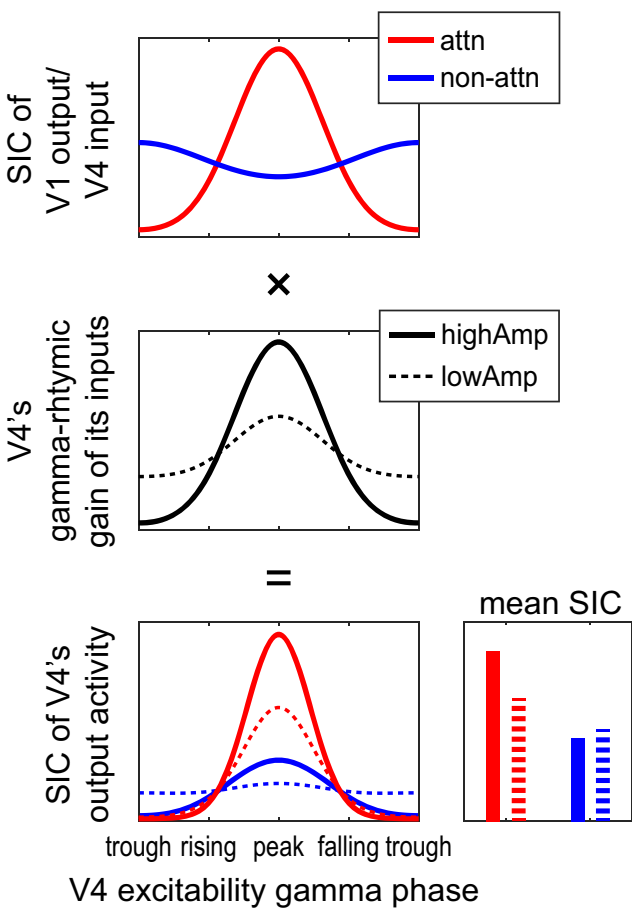

Figure 1. Routing by syncrhony mechanism predicts modulation of stimulus information content within V4's gamma-rhythmic neural activity, depending on V4's gamma-phase and amplitude. $A$, Schematic outline of the routing by synchrony (RBS) mechanism, which allows downstream neurons to process stimulus information mediated by selected subsets of afferent inputs while suppressing the information provided by other inputs. Two stimuli (red and blue shapes) compete for being processed by a downstream V4 population with a large receptive field (RF) containing both stimuli indicated by the gray dashed oval. Each visual stimulus is contained within RFs of separate V1 populations (red and blue dashed ovals) evoking spiking activity within their corresponding V1 population (red and blue vertical bars in the insets). The V1 populations exhibit gamma-rhythmic activity, causing their spikes to occur in bursts. These bursts of spikes act as input to V4, which exhibits its own gamma-rhythmic activity. The rhythmic activity of the V1 population corresponding to the attended stimulus is synchronized with V4's gamma-rhythm in a favorable phase relationship, such that its spikes arrive at V4 when it is most excitable (left inset, red arrows). This effectively evokes spiking activity in V4, resulting in a reliable transfer of the attended stimuli's information. Conversely, the rhythmic activity of the V1 population with the non-attended stimulus in its RF exhibits substantially less phase-locking with V4's gamma-rhythm (Bosman et al., 2012; Grothe et al., 2012), resulting in many cycles where the bursts of spikes arrive at V4 when it is least excitable, failing to evoke further spikes (right inset, light blue arrows). In consequence, the transfer of the non-attended stimulus signal is suppressed. $\boldsymbol{B}$, Scheme showcasing how the information contained within attended and non-attended stimuli (stimulus information content, SIC) should be modulated depending on V4's gamma-phase and amplitude in accordance with RBS. Assuming that the upstream cortical population processing the attended stimulus establishes a favorable phase relationship with V4 (as shown in $\boldsymbol{A}$, left inset), the highest amount of attended SIC should arrive during V4's excitability peak (red line in top plot). Assuming that for the non-attended stimulus, the corresponding upstream population establishes substantially less phase-locking with V4, and in a predominantly anti-phasic relationship, we expect SIC modulation to be much lower, with a slightly higher amount of non-attended SIC arriving at V4's excitability trough than at its peak (blue line in top plot). The middle plot shows V4's gammarhythmic gain (solid black line for high-amplitude gamma-activity and dashed black line for low amplitude). Modulating the phase-specific SIC inputs to V4 (top plot) by V4's gain (middle plot) provides a prediction of how attended and non-attended SIC should depend on V4's excitability gamma phase and amplitude within its output activity (bottom plot). The corresponding bar plot (bottom right) displays the average SIC within V4's output activity independent of phase, demonstrating how attended and non-attended SIC should change during V4's high-amplitude versus low-amplitude gamma-activity (solid colored bars for high-amplitude condition and dashed colored bars for low-amplitude condition).

presynaptic and postsynaptic populations (Fries, 2005, 2015; Kreiter, 2006, 2020). In this scheme, gamma-rhythmic activity serves multiple functions. For presynaptic populations, it coordinates their spiking output into periodic bursts, increasing their postsynaptic impact (Steinmetz et al., 2000; Fries et al., 2001; Azouz and Gray, 2003; Taylor et al., 2005; Zandvakili and Kohn, 2015). These periodic bursts of spikes focus stimulus information into gamma-rhythmic packages (Womelsdorf et al., 2012). For postsynaptic populations, gamma-rhythmic activity enacts modulation of gain of its inputs, with alternating windows of high and low excitability (Atallah and Scanziani, 2009; Buzsáki and Wang, 2012; Vinck et al., 2013; Salkoff et al., 2015; Ni et al., 2016). When both sending and receiving populations exhibit gamma-rhythmic activity, they can establish coherent states with coupled phases (Fig. 1A). In a favorable state for information routing, the gamma-rhythmic bursts of spikes from the presynaptic population arrive at the receiving population during windows of high excitability, resulting in enhanced information transfer. In an unfavorable phase relationship, the spikes arrive predominantly during windows of inhibition in the receiver population, resulting in suppressed information transfer. Thus, effective connectivity between presynaptic and postsynaptic populations can be modulated by establishing an appropriate phase relationship between their gamma-rhythms. This mechanism has been referred to as communication-through-coherence (CTC; Fries, 2005, 2015), or as routing-by-synchrony (RBS; Kreiter, 2006, 2020; Grothe et al., 2012, 2018; Palmigiano et al., 2017). In support, research has shown that $\mathrm{V} 4$ populations establish stronger phase coherence with the presynaptic populations processing attended stimuli as opposed to those processing non-attended stimuli (Bosman et al., 2012; Grothe et al., 2012).

Cumulatively, RBS posits that stimulus information arrives to V4 from lower visual areas in the form of gamma-rhythmic information packages, where the phase relationship between the sending and receiving populations determines whether information gets passed on or suppressed (Fig. 1B). Additionally, the gammaoscillation amplitude is not constant (Bosman et al., 2009; McLelland and VanRullen, 2016; Spyropoulos et al., 2018), so one also expects selective routing to be enhanced (or diminished) during periods of higher (or lower) amplitude gamma-activity. 
Here, we directly investigate whether selective information transfer of extended, time-varying signals in V4 complies with the RBS mechanism. For this purpose, we quantify attended and nonattended stimulus information content (SIC) within V4's neural activity at different phases and amplitudes of successive gamma-oscillation cycles. If gamma-synchronization and selective information routing were causally linked to each other, we expect SIC to be modulated by phase with its maximum corresponding to V4's excitability peaks (Fig. 1B). This effect should be consistently higher for the attended signal compared with the non-attended, increasing (or decreasing) during periods of high (or low) gamma-activity.

\section{Materials and Methods}

Experimental model and subject details All procedures and animal care were in accordance with the regulation for the welfare of experimental animals issued by the federal government of Germany and were approved by the local authorities. Data from two adult male rhesus monkeys (Macaca mulatta) were used for this study. Parts of the data have been used in a previous publication (Grothe et al., 2018).

Surgical procedures and behavioral task Details about the surgical preparation, behavioral task, and recording details have been reported previously (Grothe et al., 2012, 2018). In short, animals were implanted under aseptic conditions with a post to fix the head and a recording chamber placed over area V4. Before chamber implantation, the monkeys had been trained on a demanding shape-tracking task (Fig. 2A). Neural signals were measured from area V4 with one to three epoxy-insulated tungsten microelectrodes (125 $\mu \mathrm{m}$ in diameter; $1-3 \mathrm{M} \Omega$ at $1 \mathrm{kHz}$; Frederic Haer). Reference and ground electrodes for Monkey $\mathrm{F}$ were platinum-iridium wires below the skull at frontal and lateral sites. The reference for Monkey B was a platinum-iridium wire placed posteriorly below the skull, and the ground was a titanium pin at the posterior end of the skull.

The task (Fig. $2 A$ ) required fixation throughout the trial within a fixation window (diameter $1-1.5^{\circ}$ of visual angle) around a fixation point in the middle of the screen. Eye position was monitored at $100 \mathrm{~Hz}$ using a video-based eye tracking system (Monkey B: custom made, Monkey F: IScan Inc). If the animal moved its eyes away from the fixation point, the trial was immediately terminated. Microsaccades within the fixation window were extracted by computing eye movement speed and extracting windows where it exceeded 3 SDs of its mean.

After a baseline period, the monkeys had to covertly attend to one of two statically presented, closely spaced stimuli (shapes) that was cued (static/cue period). Then both shapes started morphing into other shapes. The monkeys were trained to respond by releasing a lever when the cued initial shape reappeared at a pseudo-randomly selected position in the shape sequence, after two to five morph cycles. The animals had to ignore reappearance of the initial shape in the distracter sequence. The shapes were placed at equal eccentricity. Stimuli were presented with a refresh rate of $100 \mathrm{~Hz}$ on a 22-inch CRT monitor containing
$1152 \times 864$ pixels (Monkey B) or $1024 \times 768$ pixels (Monkey F), which was placed at a distance of $92 \mathrm{~cm}$ (Monkey B) or $87 \mathrm{~cm}$ (Monkey F) in front of the animal.

In order to be able to track the information content of the stimuli within the neural activity, we used filled shapes and tagged the neural activity they evoke with imposed broadband luminance fluctuations ("flicker") on the stimuli: we changed the luminance of the shapes by choosing a random, integer gray pixel value with each frame update of the display. For Monkey F, the values were drawn from an interval [128, $172]$, and for Monkey B, from the full range [0, 255], corresponding to luminance fluctuations in a range of $6.9-12.5$ and $0.02-38.0 \mathrm{Cd} / \mathrm{m}^{2}$, respectively. Both shape streams had their own independent flicker time series of luminance values. Note that the flickering of the stimuli was not relevant to perform the task. A few trials were included in which only one stimulus was presented for offline controlling of response strength to individual stimuli.

\section{Recording}

The electrodes' signals were amplified by a factor of 1000 (Monkey B) or 5000 (Monkey F; MPA32I and PGA 64, 1-5000 Hz, Multi-Channel Systems $\mathrm{GmbH}$ ) and digitized at $25 \mathrm{kHz}$ (Monkey B: USB-ME-256 System, Multi-Channel Systems GmbH, Monkey F: A/D converter board, Multichannel systems and $\mathrm{C}++$ based custom-made data acquisition system). For positioning stimuli, RFs were mapped manually while the monkey was fixating centrally, followed by an automated mapping procedure consisting of rapid presentations of circular dots. Stimuli were placed at equal eccentricity in the RF 
A

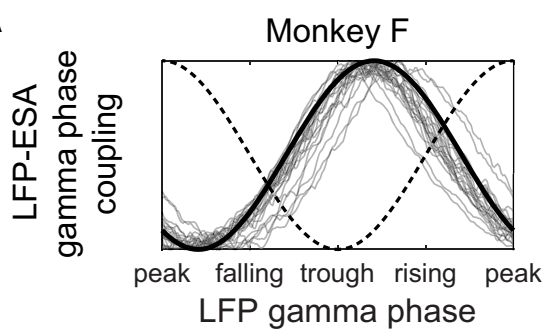

B

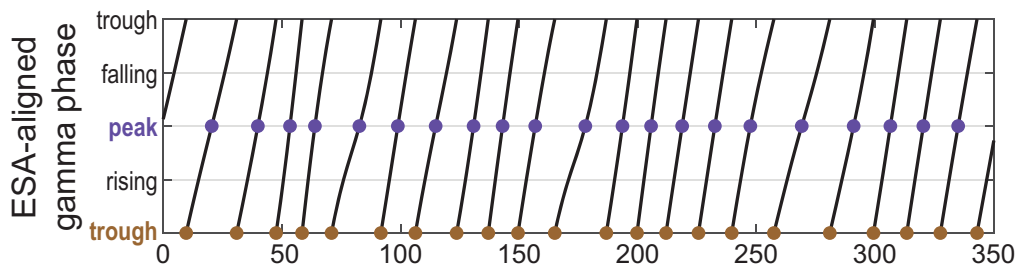

C

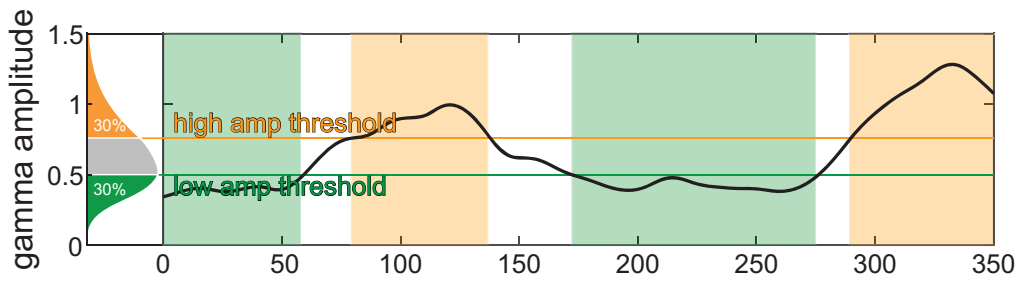

D

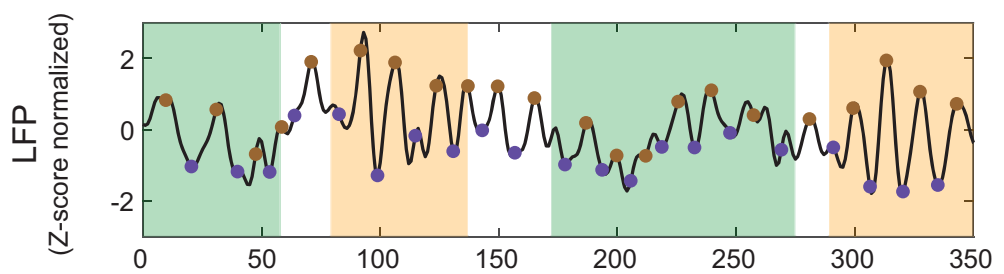

$\mathbf{E}$

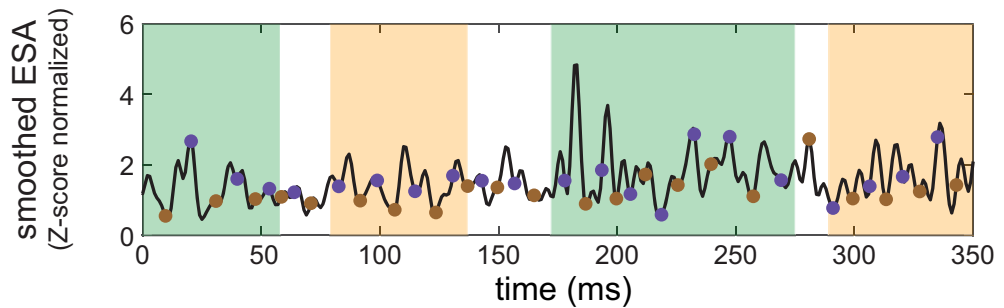

Figure 3. Extraction of gamma-phase-specific and gamma-amplitude-specific neural signals. $\boldsymbol{A}$, Normalized spike-field coupling in the gamma-frequency range, computed from ESA and LFP signals, for all the recording sites for each animal (thin lines for each site, bold line for the mean across sites). The peak of spiking activity consistently occurs at roughly the trough of LFP's gamma-cycle (indicated by dotted line), as expected for the superficial layer (van Kerkoerle et al., 2014). By shifting LFP's gamma-phase by the appropriate amount, individually for each trial set, we attain the ESA-aligned gamma-phase, which is used as a proxy for excitability gamma-phase throughout the rest of the analysis. $\boldsymbol{B}$, Time course of ESA-aligned gamma-phase corresponding to a 350-ms snippet of a trial. The locations of peaks are marked with purple dots, and excitability troughs with brown dots. C, Corresponding gamma-amplitude with 30\% highest and 30\% lowest thresholds marked with horizontal lines. The thresholds are computed from the distribution of amplitudes gathered across the whole set of trials, shown to the left of the main plot. Periods of time when the amplitude surpassed the high threshold are shaded in orange, and periods of time below the low-amplitude threshold are shaded in green. $\boldsymbol{D}$, The corresponding LFP neural activity with precise peak and trough times of the ESA-aligned gamma-phase identified in $\boldsymbol{B}$, and high and low amplitudes identified in $\boldsymbol{C}$. By using the corresponding samples of the neural activity (either purple or brown dots for peak versus trough, or orange or green time periods for high-amplitude vs low-amplitude, or a combination of both), we can compute the amount of SIC within the selected components of the neural activity. Note that the LFP is obtained by low-pass filtering the recorded signal and thus each value represents neural activity from a small time window around it. $\boldsymbol{E}$, Same as in $\boldsymbol{D}$, but for the corresponding ESA signal.

such that they induced similarly strong gamma-rhythmic activity (for details, see Grothe et al., 2018). This requirement was successfully fulfilled in 16 recording sessions, resulting in 35 recording sites in total (Monkey F: 23 sites, Monkey B: 12 sites).

Data preprocessing and site selection

All the analyses were performed using custom codes written in MATLAB. From the recorded raw data, we extracted the local field potential (LFP) as a proxy reflecting average neural activity around the recording site, and the entire spiking activity (ESA) as a measure of local spiking activity (Drebitz et al., 2018, 2019). All filters used in the process were realized as forward-backward FIR-filters to preserve the phase of the original signal, using the function "eegfilt" from EEGLab (Delorme et al., 2011) with standard parameters for the cutoffs:

- For obtaining the LFP signal, we extracted the low frequency component from the $25-\mathrm{kHz}$ raw neural recordings by applying a bandpass FIR filter with band stops at 1 and $200 \mathrm{~Hz}$.

- For obtaining the ESA signal, the raw data was first bandpass-filtered from $400 \mathrm{~Hz}$ to $2500 \mathrm{~Hz}$. We then took the absolute value of the result, and subsequently applied a second bandpass FIR filter with band stops at 1 and $200 \mathrm{~Hz}$ to simplify phase dissociation (see detailed explanation below).

Finally, both signals were downsampled to $1000 \mathrm{~Hz}$ and $z$ score normalized, yielding $y_{L F P}$ and $y_{E S A}$ used in our data analysis.

For ensuring that the recording sites were within the superficial layers of V4, we employed an additional selection criterium: The shape of the visual evoked potential (VEP) caused by the stimulus onset shows the characteristic time course expected for the superficial layers, which starts with a negative deflection as opposed to the initial positive deflection observed in the deeper layers (for details, see Givre et al., 1994; Nandy et al., 2017). We computed the VEP for each recorded site by averaging its LFP over trials. These selection criteria left 12 recording sites for Monkey $\mathrm{F}$ and 10 for Monkey B.

The experimental setup involved having two stimuli within the V4's RF; each location was cued to be attended for half of the recorded trials in each session. We decided to split the trials of each recording site by the attended location, providing us with 24 datasets for Monkey F and 20 datasets for Monkey B. This was possible because $\overline{S C}$ values from trial sets split by attended location were as statistically independent as $\overline{S C}$ values from different recording sites, suggested by the fact that the distributions of the differences between the split sets and the full sets were not significantly different (Wilcoxon signed-rank test).

\section{Data analysis}

Data analysis consisted of several processing steps applied to the LFP and ESA signals in sequence (Fig. 3). First, gamma phase and amplitude were extracted from the LFP, and gamma-phase converted to excitability phase by assessing the spike-field coupling from ESA and LFP activity. Then, gamma excitability phase was used to isolate the signal values occurring at specific phases to form a phase-specific signal. Periods of high (low) gamma-amplitude were selected to form an amplitude-specific signal. In the final step of the analysis, SIC in the neural signals was 
assessed by computing the spectral coherence between the luminance flicker and the dissociated LFP or ESA signals. In the following sections, each of those steps are explained in detail.

\section{Extraction of gamma-phase and amplitude}

By applying a wavelet transform with Morlet kernels, we computed the average power spectrum of the LFP signal $y_{L F P}$ during the period in which the stimuli were morphing until $200 \mathrm{~ms}$ before a correct response, and normalized it by the average power spectrum observed before stimulus onset in the baseline period (separately for each recorded site). The spectra revealed clear peaks in the gamma-frequency range, of which we extracted a lower and upper frequency limit by taking location at half of the highest point around the peak $(\sim 40-100 \mathrm{~Hz}$ for Monkey F recordings, and $50-110 \mathrm{~Hz}$ for Monkey B). Subsequently, gamma-activity was obtained by applying a forward-backward FIR bandpass filter to the LFP with cutoff frequencies determined by the lower/upper frequency limits. By applying the Hilbert transform to the result, we obtained LFP's gamma-phase $\Phi_{L F P}$ and amplitude $\mathrm{A}_{L F P}$.

Next, we would like to know when in each gamma-oscillatory cycle neuronal activity is maximal, as a proxy for maximal excitability. Ideally, phases of high (or low) excitability should roughly correspond to high (or low) spiking activity. Unfortunately, the recorded ESA reflects only a small number of neurons next to the recording site, resulting in a signal that is too noisy to reliably extract gamma phase and amplitude. On the other hand, while the LFP provides a clean and reliable measure of the local populations' rhythmic activity, its recording is affected by conduction delays and phase-shifts that depend on the recording electrode impedance as well as its precise location and orientation within the neural tissue (Gabriel et al., 1996; Bédard et al., 2004; Nelson et al., 2008; Bédard and Destexhe, 2012), making it a poor proxy for excitability's phase. To resolve this issue, we related gamma-phase information from the LFPs to spiking activity contained in the ESA by computing the mean ESA value for each LFP gamma-phase, thus obtaining an estimate for the spike-field coupling. By subtracting the phase for which spikefield coupling was maximal from $\Phi_{L F P}$ we acquired the ESA-aligned gamma-phase $\Phi_{E S A}$, which served as a proxy for excitability gammaphase throughout the entire analysis. For the amplitude of excitability's gamma-rhythm, we kept LFP's amplitude $\mathrm{A}_{L F P}$.

\section{Phase and amplitude dissociation, wavelet transform}

Extraction of gamma-phase-specific components of the neural recording signals $y$ was performed before using a wavelet transform $W_{f}$ to obtain a frequency-resolved neural signal representation, while dissection with respect to gamma-amplitude was performed thereafter. Applying these three operations in sequence yields the dissected and spectrally resolved neural activity $\widetilde{z}(t, f)=D_{A}\left[W_{f} \circ D_{\varphi}[y(t)]\right]$. Here, we write amplitude and phase dissection as formal operations $D_{A}$ and $D_{\varphi}$, respectively, which were realized as follows.

For performing phase dissociation, we first determined the time points $t_{k \varphi}$ at which the excitability phase passed through a desired target phase (e.g., $\varphi=0$ for peaks, or $\pi$ for troughs). These times were then used to create a new signal $D_{\varphi}[y]$ by sampling from the original signal $y(t)$ (LFP or ESA) at those points. In conjunction with integration over time during the wavelet transform, we can formally write this notching operation by using the $\delta$ distribution:

$$
D_{\varphi}[y](t):=\sum_{k} \delta\left(t-t_{k \varphi}\right) y(t) .
$$

Note that to obtain the phase-dependency curves of the SIC, we evaluated it for a finite set of gamma-phases with equal spacing. For this reason, we wanted the "notched" signal $y\left(t_{k \varphi}\right)$ to represent not only activity at exactly the time point $t_{k \varphi}$, but also in its vicinity. This was trivially the case for the LFP since it was originally obtained by low-pass filtering. For the more rapidly varying ESA, the same level of low-pass filtering was applied, to avoid missing an activation peak by notching the signal at a slightly different time.
Amplitude dissociation was realized by first obtaining the distribution of gamma-amplitudes throughout each individual recording session. From this distribution, we selected the 70th (and 30th) percentiles to use as high- (and low-) amplitude thresholds $A_{h i}$ (and $A_{\text {low }}$ ). Using these thresholds, we selected time periods exhibiting high (or low) oscillation amplitudes by means of indicator functions:

$$
\begin{aligned}
I_{h i}(t) & =\Theta\left(A(t)-A_{h i}\right), \\
I_{\text {low }}(t) & =\Theta\left(A_{\text {low }}-A(t)\right),
\end{aligned}
$$

where $\Theta$ denotes the Heaviside function. Using these indicator functions, the amplitude specific spectra $D_{A}[\tilde{y}](t, f)$ takes the form:

$$
D_{A}[\tilde{y}](t, f):=I_{h i / l o w}(t) \widetilde{y}(t, f) .
$$

If only phase dissection was performed (no amplitude selection), we used the identity function for $D_{A}$, thus $D_{A}[\tilde{y}]=\tilde{y}$, and if only amplitude dissection was performed (no phase selection), we used the identity function for $D_{\varphi}$, thus $D_{\varphi}[y]=y$.

\section{Spectral coherence}

To evaluate how much the luminance fluctuation $x(t)$ of a shape contributed to the neural activity $z(t)$, we used spectral coherence. First, we computed the spectrograms $\widetilde{x}(t, f)$ and $\widetilde{z}(t, f)$, where $f$ is the frequency and $t$ is the time, using a wavelet transform with Morlet kernels. Here, $\widetilde{z}(t, f)$ represents the neural signals which already underwent phase and/or amplitude dissociation in conjunction with the wavelet transform as described in the preceding section. The transform yields complex valued coefficients representing the amplitude and phase of the signals. By evaluating the normalized cross-correlation between $\widetilde{x}$ and $\widetilde{z}$, we obtained the spectral coherence:

$$
S C_{x z}(f, \tau):=\frac{\left|\sum_{p, t} \widetilde{x}^{*}(f, t) \widetilde{z}(f, t+\tau)\right|^{2}}{\left(\sum_{p, t}|\widetilde{x}(f, t)|^{2}\right)\left(\sum_{p, t}|\widetilde{z}(f, t+\tau)|^{2}\right)},
$$

where $\widetilde{x}^{*}$ indicates the complex conjugate of $\widetilde{x}, \tau$ is the lag between the two signals, and where the sums are performed over the population of trials $p$ included in the computation for the time points $t$ in each trial.

Because of the normalization terms in the denominator, the values of $S C_{x z}$ lie between 0 and 1 . All sums were computed over all times for which $t$ and $t+\tau$ lie within a selected time period during a trial, from the beginning of the second morph cycle until $200 \mathrm{~ms}$ before the monkey's response. Summation was performed either over all trials from all recording sites for cumulative population analyses, or over individual sets of trials from single recording sites, separated by the attended location.

Once spectral coherence is calculated, we compute the pooled value $\overline{S C}$ over a region of interest in frequency-time lag space to reduce a twodimensional result to a single value. The region of interest was defined as a frequency-dependent cone of width $\pm \frac{7}{6} T$ around $\frac{1}{2} T+t_{\text {onset }}$, where $T=1 / f$ and $t_{\text {onset }}$ denotes the onset delay of the neural response in V4 after stimulus onset which was $50 \mathrm{~ms}$ in Monkey F and $60 \mathrm{~ms}$ in Monkey B (Grothe et al., 2018). We first took the average across lags within the frequency-dependent region of interest and then took the mean of the time averages from 5 up to $15 \mathrm{~Hz} ; 15 \mathrm{~Hz}$ was selected as upper the limit, since the majority of the individual sets results did not yield significant spectral coherence above this value.

\section{Confidence intervals and statistical tests}

For assessing significance of each $\overline{S C}$ measure, we computed the $95 \%$ chance level of its value being different from 0 (Figs. $4 A, 6 A, 7$, gray shading toward the bottom of each plot). This was done by taking the 95th percentile from the distribution of $\overline{S C}$ measurements gathered by 
pairing the neural recording signal with 200 surrogate luminance flicker signals.

95\% confidence intervals for $\overline{S C}$ were gathered by bootstrapping across trials. From a set $S$ of $N$ trials, we randomly sampled trials with replacement, generating another set $S^{*}$ with the same number of trials $N$, many of which are duplicates of each other. Using this new data, we calculated our desired test statistic $\overline{S C}^{*}$. This procedure was repeated 20,000 times, creating a distribution of $\overline{S C}^{*}$ values, from which we extracted the $2.5 \%$ and $97.5 \%$ quantiles.

In order to assess whether a test statistic $\overline{S C}_{1}$ (computed from data extracted from set $S_{1}$ with $N_{1}$ trials) is significantly different from $\overline{S C}_{2}$ (computed from set $S_{2}$ with $N_{2}$ trials), a non-parametric permutation statistical test was used (Maris and Oostenveld, 2007; Maris et al., 2007). By randomly shuffling the trials between the two sets, we created new sets $S_{1}^{*}$ with the same number of trials $N_{1}$ and $S_{2}^{*}$ with $N_{2}$ trials, each containing no trial duplicates but rather a mixture of trials from the original sets, which are then used to compute the test statistic $\overline{S C}_{1}^{*}-\overline{S C}_{2}^{*}$. This procedure was repeated 20,000 times, generating a null distribution for the test statistic, allowing to calculate the significance level $p$ value by evaluating the proportion of this distribution that fell above/below the true value of the statistic, $\overline{S C}_{1}-\overline{S C}_{2}$, computed from the original datasets. In cases where $100 \%$ of the null distribution is above/below the true statistic, the $p$ value is reported as $p<1 / 20,000$.

To assess whether a distribution of $\overline{S C}$ measures is significantly different between two conditions (i.e., whether the data clouds in the scatter plots in Figs. $4 B, 6 B$ lie above or below the diagonal), we determined whether the ratios between the $\overline{S C}$ values for individual sets are significantly different from 1 by using the Wilcoxon signed-rank test on the distribution computed via $\log \left(\overline{S C}_{1} / \overline{S C}_{2}\right)$.

\section{Results}

For investigating whether and how attention-dependent signal transfer is affected by V4's gamma-phase and amplitude, we analyzed LFPs and multiunit spiking activity (measured as ESA) recorded from the superficial layers of area V4 in two macaque monkeys (Macaca mulatta). During recording, the animals were engaged in a demanding shape tracking task requiring the monkeys to attend to one of two concurrently presented dynamic stimuli within the recorded population's RF (for details, see Fig. $2 A$ and Materials and Methods). The two stimuli consisted of complex shapes, which, after an initial static period, morphed through a series of different shapes throughout the trial. At the beginning of each trial, one of the two stimuli was cued. The task for the monkey was to attend to the cued stimulus while maintaining fixation, and to respond when its initial shape reappeared in the morphing sequence. The other, non-attended stimulus had to be ignored. The number of morph-cycles that the stimuli went through before returning to the initial shape was randomized. The neural signals included in this analysis were taken from the start of the second morph cycle until $200 \mathrm{~ms}$ before a correct behavioral response. The first cycle was excluded since it never morphed into the target shape and thus would not require the animal to pay full attention to the target shape in this particular time interval.

Crucially, the two stimuli were tagged by independent and behaviorally irrelevant random luminance fluctuations, with a luminance change every $10 \mathrm{~ms}$. This allows us to evaluate SIC in V4 activity by computing the spectral coherence between the neural activity and the luminance signals (Fig. $2 B$ ). Spectral coherence provides a frequency-resolved and time delay-resolved correlation measure between two signals. By pooling across relevant lag and frequency bins, we acquired a single value $\overline{S C}$ as a measure for the average SIC of the shapes' luminance fluctuation within the recorded neural activity.
In order to probe whether a potential SIC modulation is aligned to excitability phase as predicted in Figure $1 B$, we employed ESA as a proxy for excitability. Specifically, we first identified which LFP gamma-phase was associated with maximum ESA, and then shifted the LFP gamma-phase by the appropriate amount for each recording site giving us the ESA-aligned gamma-phase. Throughout the whole analysis, this is the gamma-phase employed as a proxy for excitability's gammaphase (Fig. 3A).

To quantify SIC in dependence on gamma phase and amplitude, we extracted neural activity specific to each phase of the gamma-cycle and separately for periods of high and low gammaband amplitudes. We then computed $\overline{S C}$ between these phase and amplitude-specific signals and the luminance fluctuations of the attended and non-attended stimuli (for details, see Fig. 3 and Materials and Methods).

\section{Gamma-phase modulates signal information content of the attended stimulus}

We extracted components of the neural signals associated with a specific gamma-phase by selecting the discrete time points that correspond to that particular phase and sampled the neural signals at those points. In the example shown in Figure $3 B$, we marked the time points corresponding to ESA-aligned gammapeaks (in purple) and troughs (in brown). The dots in Figure $3 D$, $E$ indicate which samples from the LFP and ESA signals, respectively, were obtained when selecting at peaks (in purple) or troughs (in brown). The method is not limited to sampling just from the peak or from the trough, allowing to extract a signal specific to any desired phase. By computing $\overline{S C}$ between the phase-specific neural activity signals and the input stimuli, we can assess the amount of attended or non-attended SIC in dependence on gamma-phase.

For the attended stimulus (Fig. $4 A$, left two columns), SIC at peaks $\left(\overline{S C}_{\text {peak }}\right)$ was significantly larger than SIC at troughs $\left(\overline{S C}_{\text {trough }}\right)$, consistently across monkeys and signals $(p<0.00005$ for both animal's LFP signals, $p=0.0002$ for Monkey F ESA, $p=0.003$ for Monkey B ESA, non-parametric permutation test). In contrast, for the non-attended stimulus, there was no significant difference between SIC at peaks and troughs for ESA. The LFP showed a significantly higher SIC at peak versus trough for Monkey B ( $p=0.0021$ for Monkey B LFP). The absolute modulation strength, calculated via $\overline{S C}_{\text {peak }}-\overline{S C}_{\text {trough }}$, is significantly higher for the attended stimulus than for the non-attended stimulus for both animals and neural signal types $(p<0.00005$ for both animal's LFP, $p=0.0045$ for Monkey F ESA, $p=0.0094$ for Monkey B ESA). Aside for a few values near the ESA-aligned gamma-trough for Monkey B's non-attended ESA results, all observed $\overline{S C}$ values were significantly greater than chance level, indicating that information might be transferred during all gamma-phases, although to different extents. For Monkey B, the $\overline{S C}$ values for the attended signal are significantly higher than their non-attended counterpart across all phases $(p<0.00005)$. For Monkey F, the difference between attended and nonattended conditions is significant at the peaks $(p<0.00005)$. At the falling phase for Monkey F LFP and at the trough phase of the Monkey F ESA conditions, the non-attended $\overline{S C}$ values are slightly higher than the attended ones, but after correcting for multiple comparison across all the phases, this effect is not significant.

The effect of a higher SIC at peaks than at troughs for the attended stimulus was consistent across individual recording trial sets. The scatter plots in the two leftmost columns of Figure $4 B$ 
A attended monkey $\mathrm{F}$
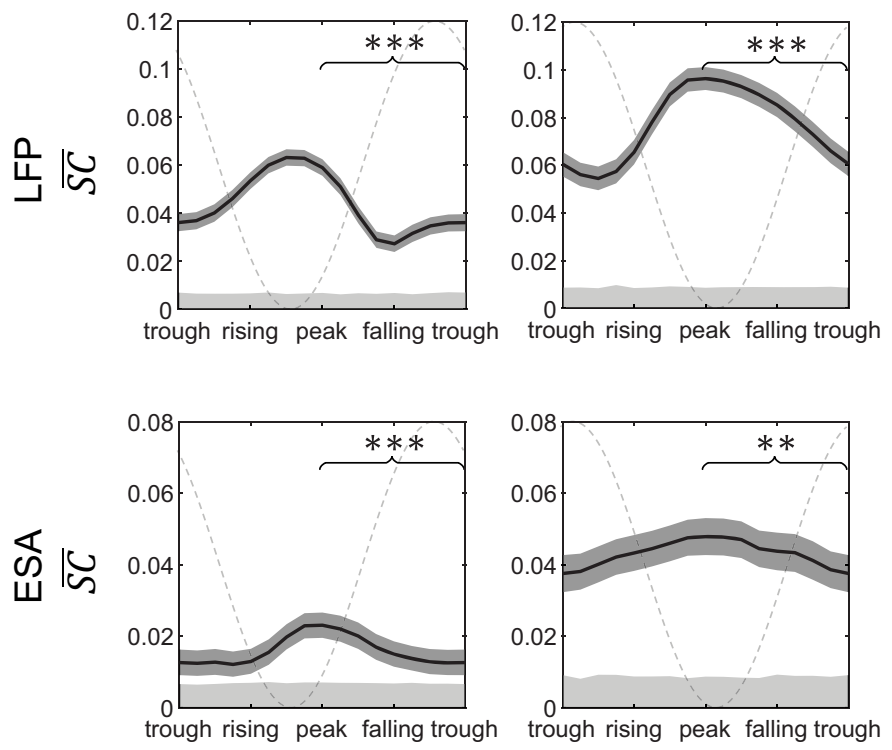

ESA-aligned gamma phase
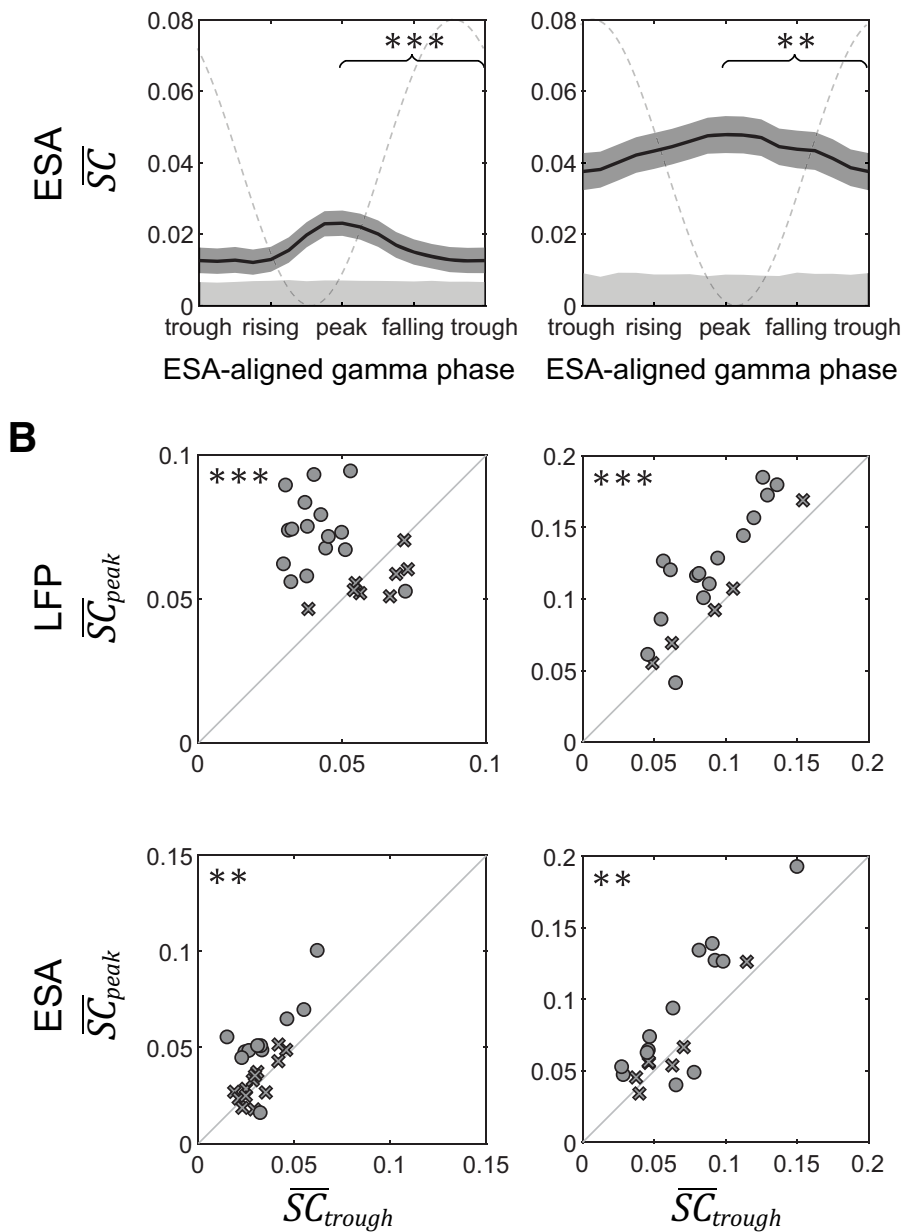

non-attended

monkey $\mathrm{F}$
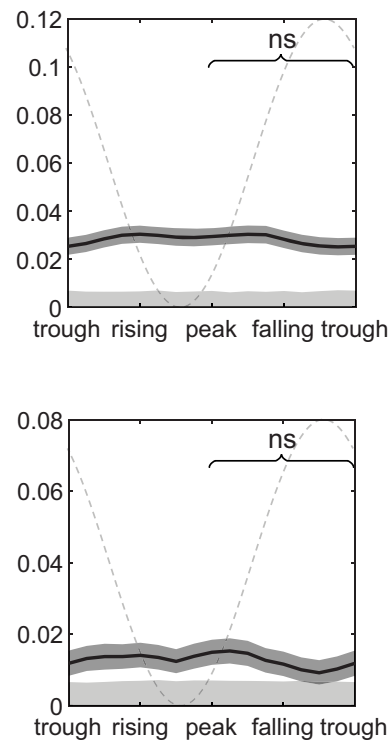

ESA-aligned gamma phase
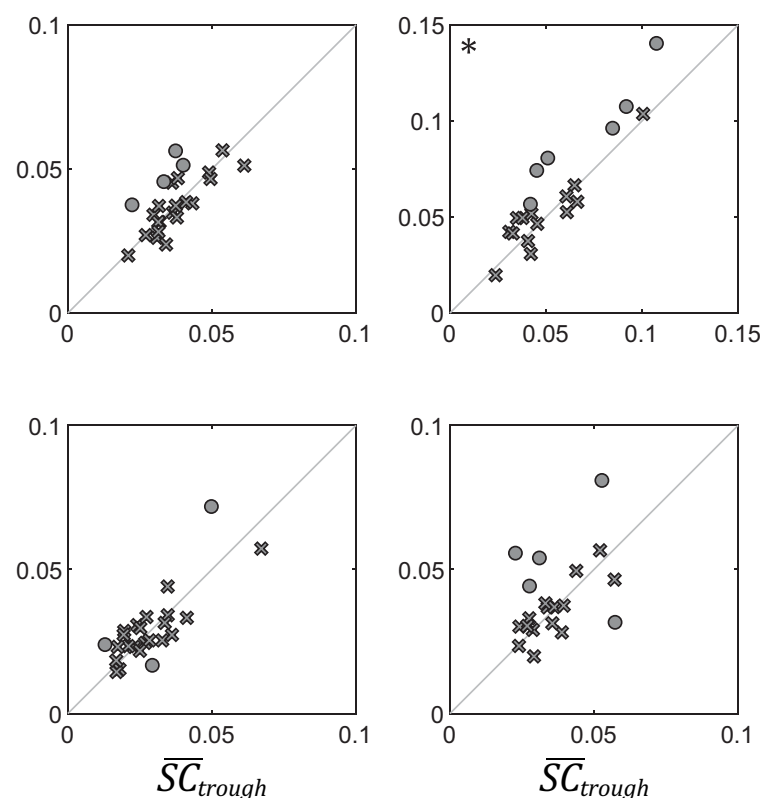

monkey B

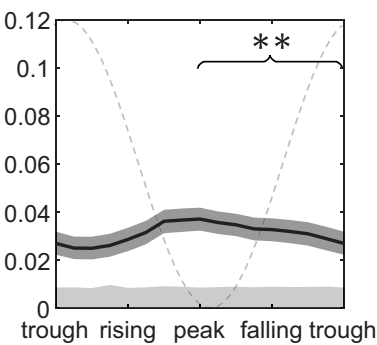

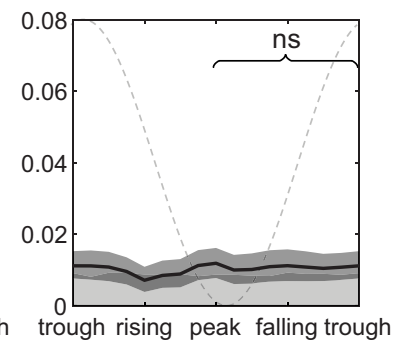

ESA-aligned gamma phase

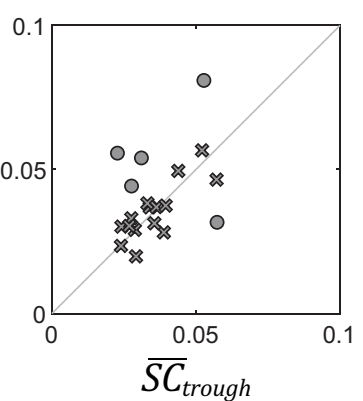

Figure 4. Stimulus information content is modulated by V4's gamma-phase. $\boldsymbol{A}$, SIC dependence on phase for data pooled across all sessions. In each plot, we display how $\overline{S C}$ depends on the ESAaligned gamma-phase (horizontal axis) from which the neural signal is extracted. The shading around each line corresponds to the $95 \%$ confidence interval. Significance of the difference between $\overline{S C}_{\text {peak }}$ versus $\overline{S C}_{\text {trough }}$ is indicated in each plot ( $\mathrm{ns}=$ not significant, $* p<0.05, * * p<0.01, * * * p<0.001$ ). The gray shading at the bottom of each plot corresponds to the $95 \%$ chance level (values below this level indicate no significant SIC). The gray dashed sinusoid line indicates the corresponding average LFP phase. $\boldsymbol{B}$, SIC at peaks versus troughs of the ESA-aligned gamma-phase for individual sets. For each condition, we display a scatter plot of $\overline{S C}_{\text {peak }}$ versus $\overline{S C}_{\text {trough }}$ pairs. In the scatter plots, individual sets that exhibit a significant difference $(p<0.05)$ are marked with a circle, and with a cross otherwise. The significance of the group distribution, i.e., whether it lies significantly below or above the diagonal, is marked with black asterisks on the side that contains significantly more trial sets $(* p<0.05, * * p<0.01, * * * p<0.001)$.

display $\overline{S C}_{\text {peak }}$ versus $\overline{S C}_{\text {trough }}$ for each individual trial set for the attended stimulus. The majority of data points show significantly higher SIC at peaks rather than troughs $(p<0.05$, marked with circles; 15 of 24 for Monkey F LFP, 14 of 20 for Monkey B LFP, 10 of 24 for Monkey F ESA, and 11 of 20 for Monkey B ESA). Note that only a few trial sets exhibit significantly lower SIC at gamma-peaks (one of 24 for Monkey F LFP, one of 20 for Monkey B LFP, one of 24 for Monkey F ESA, two of 20 for Monkey B ESA).
We assessed the individual set's group statistics by determining whether the distribution of log-ratios, $\log \left(\overline{S C}_{\text {peak }} / \overline{S C}_{\text {trough }}\right)$, is significantly higher or lower than 0 , indicated by the black asterisks in the scatter plots of Figure $4 B$ (Wilcoxon signed-rank test). For the attended stimulus, the peak SIC was significantly higher than the trough SIC across both monkeys and both neural signals $(p=0.0006$ for Monkey F LFP, $p=0.0007$ for Monkey B LFP, $p=0.0062$ for Monkey F ESA, and $p=0.0089$ for Monkey B ESA). For the non-attended stimulus, the scatter plots show a 


\section{bandpass center frequency}
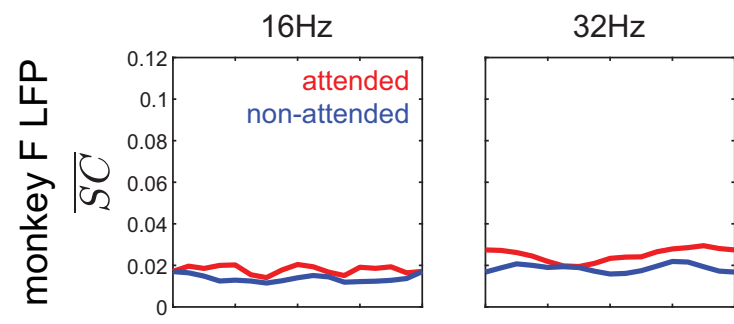

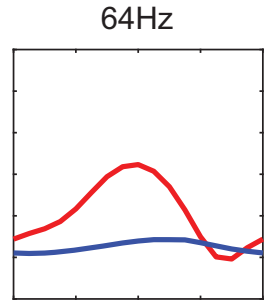

$128 \mathrm{~Hz}$
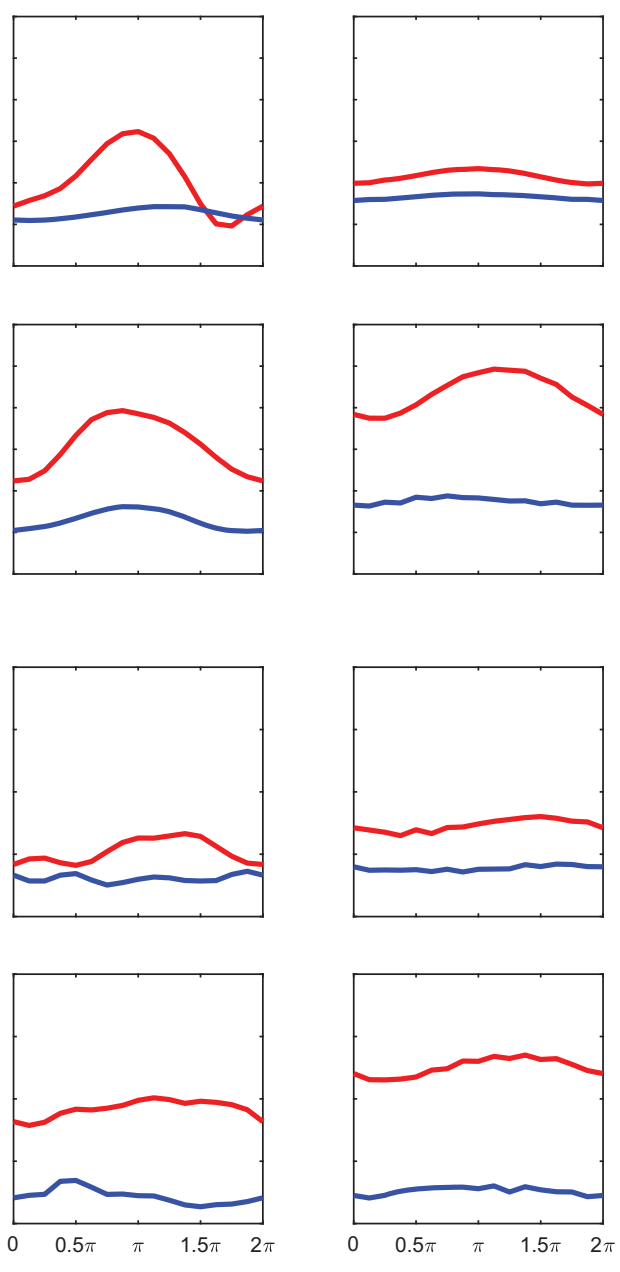

LFP phase
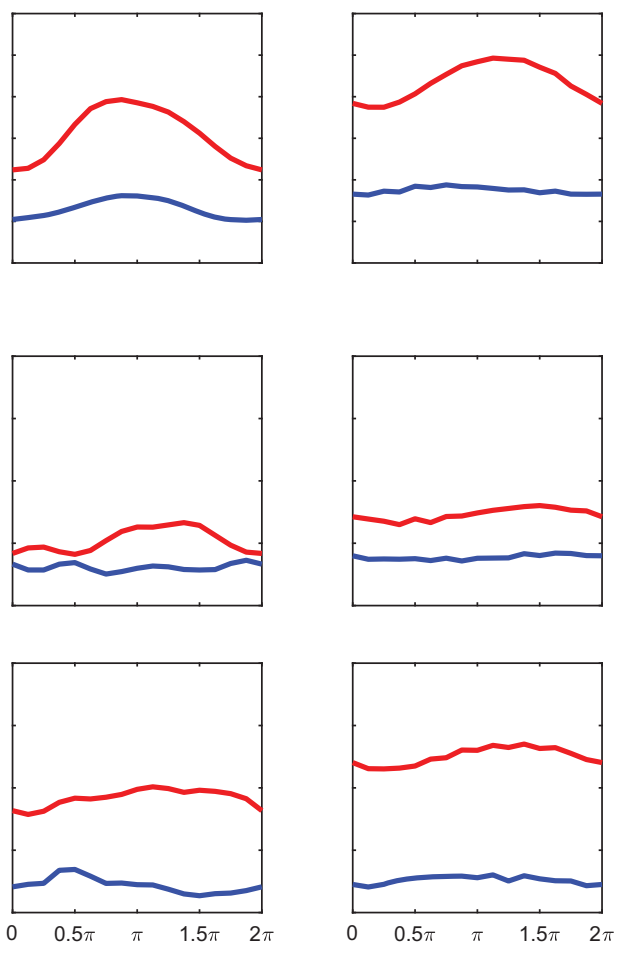

LFP phase
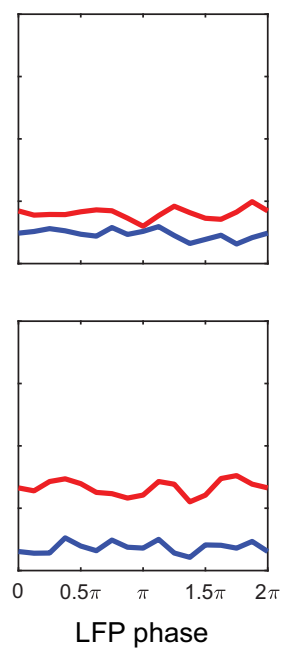

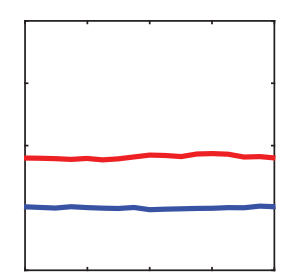

$256 \mathrm{~Hz}$
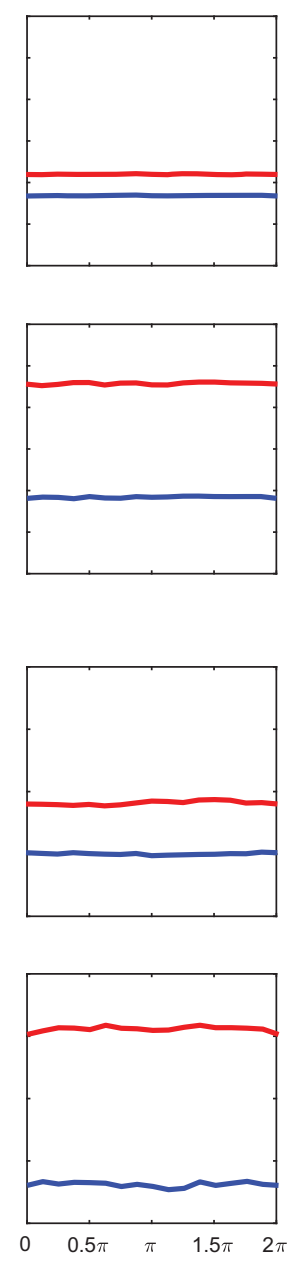

LFP phase

Figure 5. Stimulus information content modulation by phase for multiple frequency bands. SIC dependence on phase for data pooled across all sessions, with the phase-specific neural activity extracted in the same manner as before, but using phase computed from LFP bandpassed across multiple frequency bands. The horizontal axis denotes LFP phase with $\pi$ corresponding to signal troughs, which, in the case of the gamma-band (center column), should roughly correspond to excitability peaks.

tendency to exhibit higher $\overline{S C}_{\text {peak }}$ than $\overline{S C}_{\text {trough }}$ with more significant results above the diagonal, however, the group statistic was only significant for Monkey B's LFP ( $p=0.0145$, Fig. $4 B$, top right scatter plot).

In order to confirm that the phase modulation effects are specific to the gamma-band, SIC modulation by phase was analyzed across multiple frequency bands for both LFP and ESA signals (Fig. 5). This was done using the very same procedure as for the main analysis, but using phase computed from LFP bandpassed with different center frequencies. This control analysis revealed that the phase-modulation effect is indeed strongest for the gamma-band.

\section{Gamma-amplitude modulates signal information content of the attended stimulus}

For the amplitude dissection, we first gathered the distribution of gamma-amplitudes throughout each individual trial set. From this distribution, we selected the 70th (and 30th) percentiles to use as high (and low) cutoff thresholds to select activity from high and low gamma-amplitude periods. Using these thresholds, for each trial, we selected the time periods exhibiting high (or low) oscillation amplitudes. In the examples in Figure $3 C$, the corresponding periods are indicated by orange and green shading, respectively. In Figure $3 D, E$, the same shading indicates which periods of the LFP and ESA signals were selected by amplitude dissociation. Since the thresholds were computed across a set of trials, the total proportion of each individual trial included in the analysis varied slightly, with most trials' proportion falling within the $20-40 \%$ range. On average, the duration of each individual period was around $40 \mathrm{~ms}$ with $90-\mathrm{ms}$ intervals in-between, corresponding to approximately eight periods occurring every second. Using these amplitude-specific periods, we evaluated and compared the amount of SIC within high-amplitude $\left(\overline{S C}_{\text {highAmp }}\right)$ versus low-amplitude gamma-oscillations $\left(\overline{S C}_{\text {lowAmp }}\right)$.

When we ran the analysis on a cumulative set of all the trials over all recording sites for the attended stimulus, we found a small increase of SIC in neural activity during high-amplitude gamma-oscillations in comparison to low-amplitude gamma-activity (Fig. 6A). Aside for Monkey F's LFP, the difference between the amplitude conditions is significant $(p=0.00005$ for Monkey B LFP, $p=0.0229$ for Monkey F ESA, $p=0.0034$ for 
A

\section{attended}

monkey $\mathrm{F}$
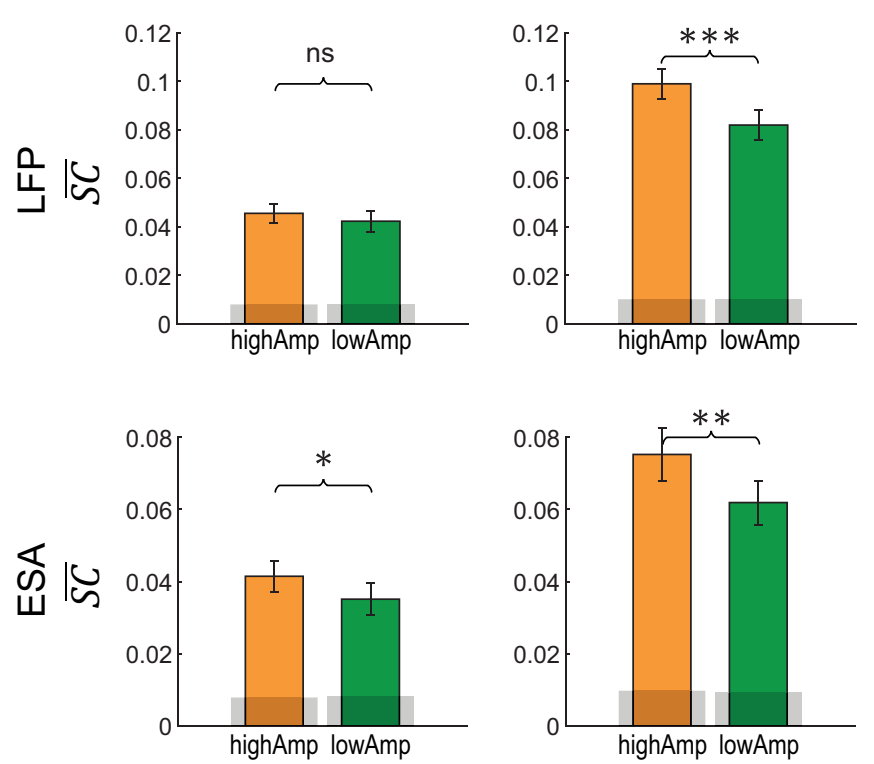

B
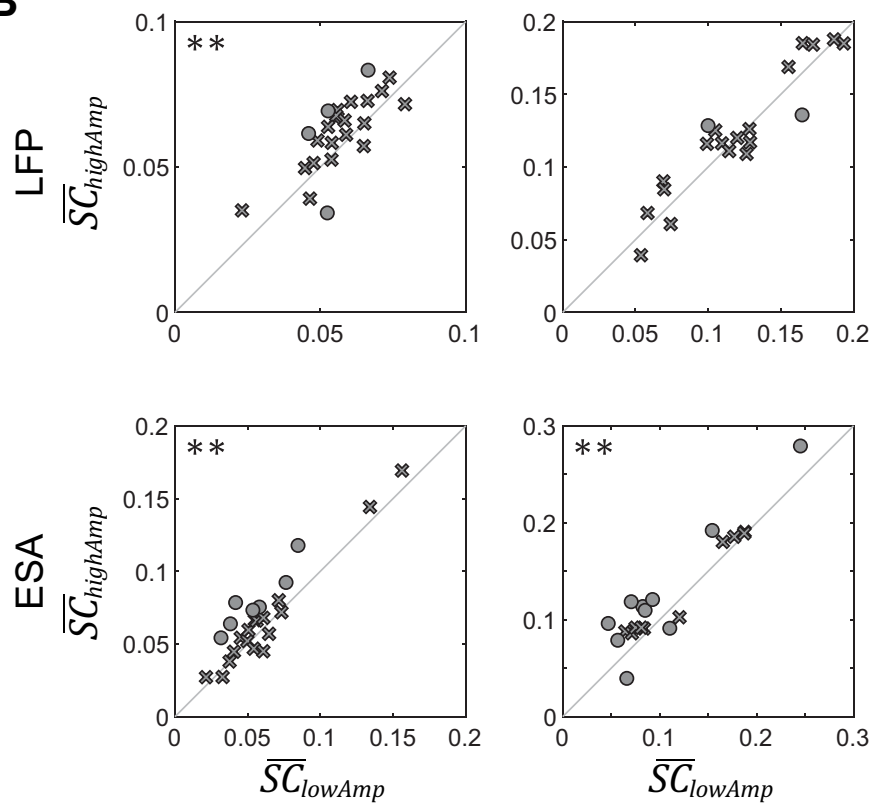

monkey B

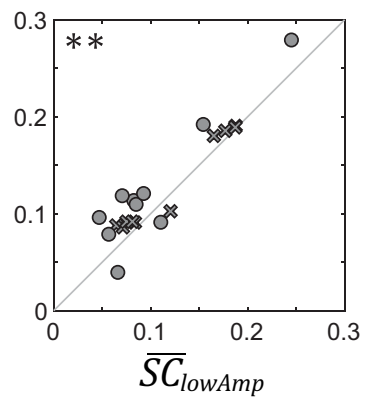

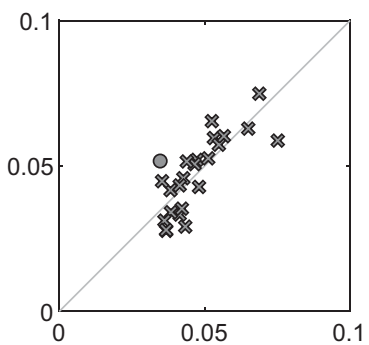

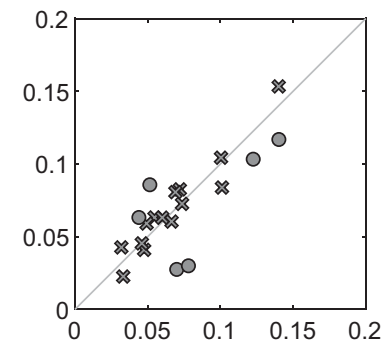

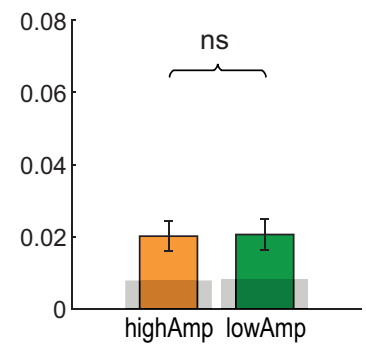

monkey $\mathrm{F}$
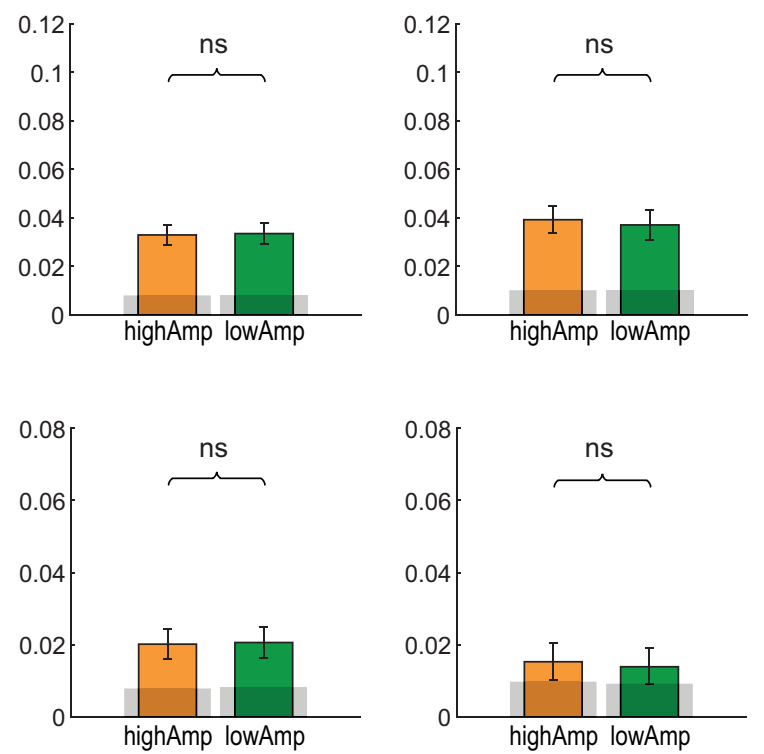

non-attended

Figure 6. Stimulus information content is modulated by V4's gamma-amplitude. $\boldsymbol{A}$, SIC extracted from periods with high-amplitude versus low-amplitude gamma-0scillations for data pooled across all recording sessions. In each plot, for the specific condition as indicated by the row and column labels, we display pairs of $\overline{S C}_{\text {highAmp }}$ (in orange) and $\overline{S C}_{\text {lowAmp }}$ (in green), separately for each animal, neural data type, and attention condition. The error bars indicate the $95 \%$ confidence interval. The gray shading at the bottom of each bar indicates the $95 \%$ chance level for that value. Significance level of the differences is indicated above each pair of bars, computed via permutation testing across the trials (ns $=$ not significant, $* p<0.05$, $* * p<0.01$, $* * * p<0.001)$. B, SIC extracted from periods of high versus low gamma-amplitude activity for individual sets. For each condition, we display a scatter plot of $\overline{S C}_{h i g h A m p}$ versus $\overline{S C}_{\text {lowAmp }}$ pairs. In the scatter plots, sets that exhibit a significant difference $(p<0.05)$ are marked with a circle, and with a cross otherwise. The significance of the group distribution, whether it lies significantly below or above the diagonal, is marked with black asterisks on the side that contains significantly more sets (ns $=$ not significant, $* p<0.05, * * p<0.01, * * * p<0.001$ ). ns, not significant.

Monkey B ESA). This corresponds to the prediction derived from our hypothesis (compare Fig. 1B). For the non-attended stimulus, the analysis did not reveal any significant differences between SIC within periods of high versus low gamma-activity.

Results from analyzing individual sites corroborated the cumulative outcomes (Fig. 6B). There are few individual trial sets that showed a significant difference because of the smaller size of the effect. For the attended stimulus, the majority of the sites with a significant difference did indicate increased information present during periods of high-amplitude oscillations (Figure $6 B$, scatter plots in the left two columns). When we looked at the distribution of the ratios $\log \left(\overline{S C}_{\text {highAmp }} / \overline{S C}_{\text {lowAmp }}\right)$, except for LFPs from Monkey B, all conditions exhibited a significant shift toward a higher SIC for high-amplitude gamma, as indicated by 
attended
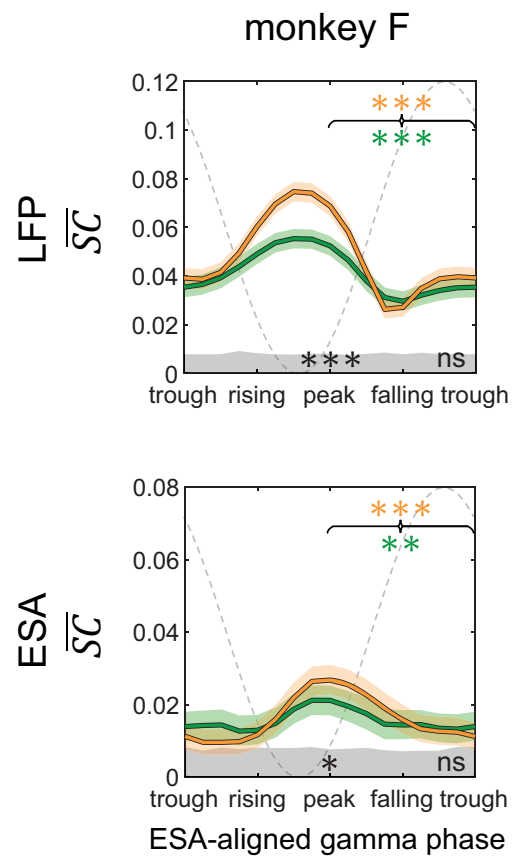
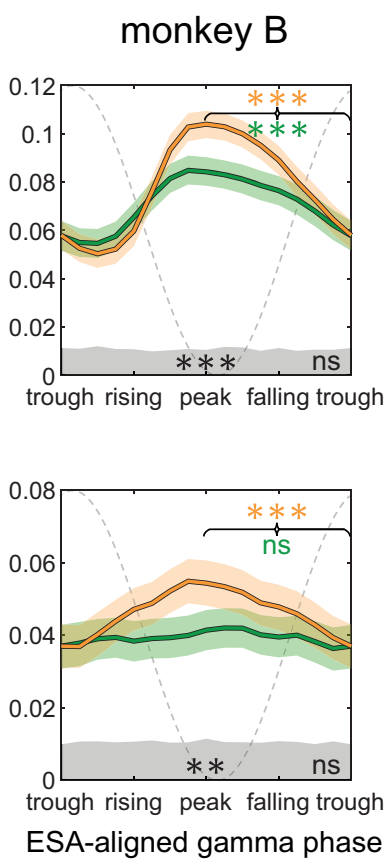

non-attended
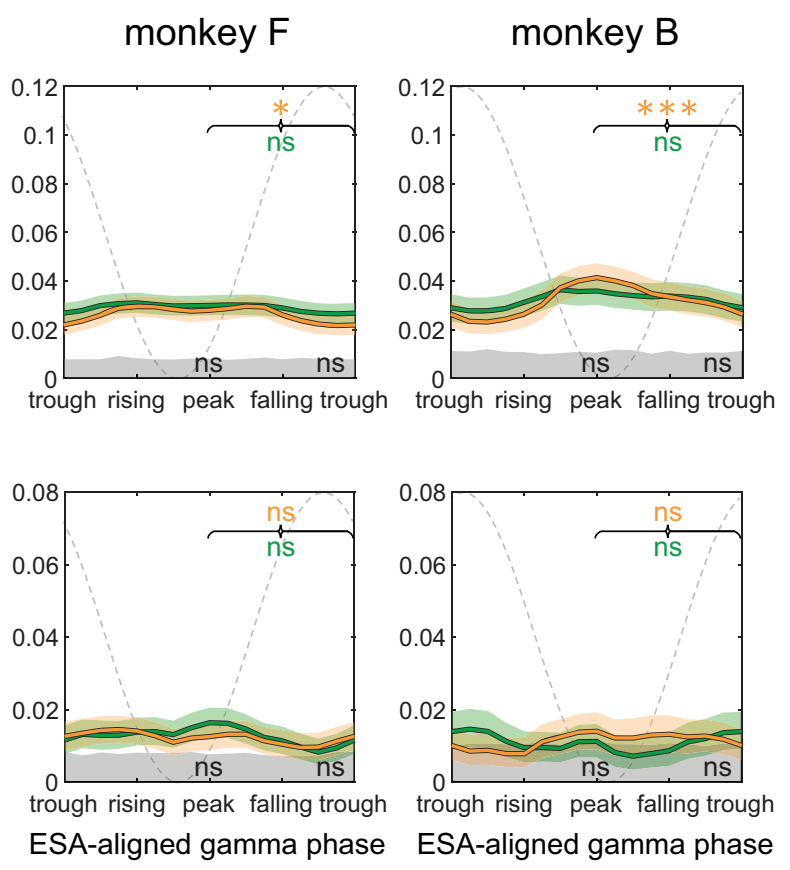

Figure 7. Signal information content during high and low gamma-amplitude periods in dependence on V4's gamma-phase. For each condition, as indicated by the row and column labels, each plot displays how SIC is modulated by phase from which the neural signal is extracted from (horizontal axis), separately for high gamma-amplitudes (in orange) and low gamma-amplitudes (in green) conditions. The shading around each line corresponds to the $95 \%$ confidence interval. The gray shading at the bottom of each plot shows the corresponding $95 \%$ chance level. The gray dashed sinusoid line indicates the corresponding average LFP phase. The asterisks toward the bottom of each plot indicate whether there is a significant difference between $\overline{S C}_{\text {highamp }}$ and $\overline{S C}_{\text {lowAmp }}$ for the peak or the trough. The colored asterisks at the top right of each plot indicated whether there is a significant difference between $\overline{S C}_{\text {peak }}$ and $\overline{S C}_{\text {trough }}$ for the corresponding amplitude condition. (ns $=$ not significant, $* p<0.05, * * p<0.01, * * * p<0.001)$.

the black asterisks ( $p=0.0057$ for Monkey F LFP, $p=0.0032$ for Monkey F ESA, $p=0.0053$ for Monkey B ESA).

For the non-attended stimulus (Fig. $6 B$, right two columns), SIC did not show any significant differences for the individual sets, except for Monkey B's ESA signal. This was the only case for which we found that $\overline{S C}_{\text {highAmp }}$ is significantly lower than $\overline{S C}_{\text {lowAmp }}(p=0.0047)$.

\section{Stimulus information content modulation by phase is increased during higher gamma-amplitude activity}

Selections of the neural activity with respect to phase and amplitude were combined to acquire LFP and ESA activity associated to the co-occurrence of a particular gamma phase and amplitude (Fig. 3).

In Figure 7, the results for the SIC computed from the set of all trials collected from all the recording sites is displayed, with the high-amplitude results in orange and low-amplitude results in green. Corroborating the hypotheses in Figure $1 B$, the data exhibited higher SIC modulation by gamma-phase within highamplitude periods and lower SIC modulation by phase for the low-amplitude periods.

The outcome lends itself to multiple tests: comparing SIC at high amplitude versus low amplitude at peak and trough (significance indicated at the bottom of each plot at peak and trough phases in black) and comparing SIC at peak versus trough phases in either high-amplitude or low-amplitude conditions (significance indicated at the top of the plot with colors corresponding to the amplitude conditions).

The difference between high amplitude and low amplitude for the attended stimulus was strongly phase-specific: while SIC near peaks within high gamma-amplitude activity was significantly larger than SIC within low gamma-amplitude activity $(p<0.00005$ for Monkey F and Monkey B LFP, $p=0.0314$ for Monkey F ESA, $p=0.0020$ for Monkey B ESA), there were no significant differences near troughs. Consequently, SIC modulation by gamma-phase was higher in the high-amplitude condition $\left(\overline{S C}_{\text {peak }}\right.$ is greater than $\overline{S C}_{\text {trough }}$ with $p<0.00005$ for all datasets), and lower (but still significant in three out of four cases) in the low-amplitude condition $(p<0.00005$ for Monkey F and Monkey B LFP, $p=0.009$ for Monkey F ESA and not significantly different for Monkey B LFP). The absolute modulation strength, $\overline{S C}_{\text {peak }}-\overline{S C}_{\text {trough }}$, is significantly larger for the attended signal versus the non-attended signal in the high-amplitude condition $(p<0.00005$ for both animal's LFP, $p<0.00005$ for Monkey F ESA, $p=0.0025$ for Monkey B ESA) and decreases for the low-amplitude condition (still significant with $p<0.00005$ for both animals' LFP, not significant for ESA). Overall, this corroborates the prediction from the corresponding curves in Figure $1 B$ for the attended signal, SIC modulation by phase is increased during high-amplitude gamma-activity and decreased during low-amplitude gamma-activity.

Similar to the previous phase-only analysis (compare Fig. 4), SIC modulation by phase was reduced dramatically for the nonattended stimulus when compared with the attended stimulus (Fig. 7, right two columns). However, in the high-amplitude condition, we observed more cases with a significantly higher SIC at the peak versus the trough $(p=0.0274$ for Monkey F LFP, $p=0.0003$ for Monkey B LFP). In the low-amplitude condition, the differences between peaks and troughs became insignificant. There were no significant differences between the high-amplitude and low-amplitude conditions at any phase. 


\section{Eye movements do not explain modulation of stimulus} information content by gamma phase and amplitude

Throughout the recording sessions, if the animal moved its eyes away from the fixation point, the trial was immediately terminated and subsequently excluded from the analyses. However, there were still microsaccades within the close vicinity of the fixation point occurring at a rate of $0.82 \mathrm{~Hz}$ for Monkey $\mathrm{F}$ and $0.77 \mathrm{~Hz}$ for Monkey B (on average across the recording sessions). Microsaccades have been previously reported to modulate firing rate (Leopold and Logothetis, 1998) and gamma-band activity in V4 within a 400-ms period following each microsaccade (Bosman et al., 2009). In order to account for any possible effects of microsaccades, we ran a control analysis, repeating the entire procedure on the data set excluding 400-ms periods following every microsaccade.

Furthermore, we detected small but significant differences in the overall distribution of eye positions within the fixation window, between attended versus non-attended conditions, as well as between the periods corresponding to high versus low gammaamplitudes. These differences could potentially lead to a different coverage of the stimuli by the V4 RFs, possibly affecting SIC of the population's activity. To address this issue, we ran a second control, repeating the analysis on a subset of data by removing periods of data until a Kolmogorov-Smirnoff test was unable to detect a difference in the corresponding distributions of eye position $(p>0.1)$.

The results from both control analyses revealed almost identical SIC measurements and significance test results (data not shown) compared with the ones presented in Figures 4, 6, 7. Throughout all the test results, the only notable change happened for the small difference between SIC at peak versus SIC at trough for the non-attended signal in Monkey F's LFP activity: the significance of the difference in Figure $4 A$, top plot in third column, changes from $p=0.0587$ to $p=0.0245$ becoming significant in the eye-position control analysis, and changes from $p=0.0274$ to $p=0.158$ becoming not significant for the highamplitude condition in Figure 7 when performing the microsaccade-control analysis. These findings indicate that the results of our analyses are not confounded by eye movements.

\section{Discussion}

In the present work, we tested the prediction of the RBS mechanism that attention-dependent visual stimulus information is not continuously dispersed over time but instead occurs selectively in pulsed information packages, modulated by phase and amplitude of V4's gamma-rhythmic activity.

Our analysis reveals that the luminance fluctuations tagging the attended stimulus are expressed most strongly within neural activity close to the phase in the gamma-oscillation cycle where local spiking peaks (i.e., close to the LFP troughs), showcasing a pattern of modulation significantly different from the nonattended signal. During periods with high-amplitude gammaoscillations, the overall SIC for attended stimuli is higher than during low-amplitude oscillations, whereas for non-attended stimuli, there is no significant difference. SIC's increase with amplitude is particularly strong at the peak of spiking activity and absent at the trough. Since the peaks of spiking activity should roughly correspond to the peaks of excitability, these results corroborate central predictions derived from the RBS mechanism.

Certain details of the results seem to deviate from the predictions illustrated in Figure $1 B$, bottom plot. For instance, when comparing attended and non-attended SIC, our expectation was that the non-attended SIC should be at least as large as the attended SIC in the vicinity of excitability troughs. However, for
Monkey B, we find that the attended signal is consistently better expressed across all phases. This may be explained by a temporal dispersion of information across phase, which occurs because of multiple factors. First, the precision of phase estimation itself is limited by noise in the recorded neural activity. Additionally, we also smoothed the neural activity before the phase-specific signal extraction, such that the value of the signal at each time point represents a temporal window of activity around it. Finally, computing SIC based on spectral coherence involves comparing the neural recording and luminance fluctuation signals with wavelets that are centered at the time of interest but also extend in temporal space. Taken together, these methodological limitations all lead to the luminance flicker signal being partially mapped to phase ranges at which it does not actually occur, thereby increasing SIC in low-SIC ranges and decreasing SIC in the high-SIC ranges of the oscillatory cycle. In consequence, our analysis is likely to underestimate the magnitude of SIC modulation by phase.

Dissociating signal content with respect to gamma-amplitude could be subject to a similar reduction in SIC modulation amplitude. If we assume constant contributions of noise throughout the recordings, precision of phase estimation will decrease during periods of low gamma-amplitude activity. Such an effect may contribute to the differences between high-amplitude and low-amplitude SIC results observed in Figure 7, where we perform a simultaneous phase and amplitude-specific signal extraction.

The results for the non-attended signal provide further insight into the details of the RBS mechanism. Here, we observe only weak, or no phase-dependent modulation of SIC. The absence of a strong modulation implies a certain coherence for the non-attended signal with the receiving population in an antiphasic relationship to counteract V4's gain modulation. This is compatible with a weak level of coherence between non-attended V1 and V4 as observed in Grothe et al. (2012). On the other hand, a weak modulation of non-attended SIC in V4 could emerge if there is no coherence between non-attended V1 and V4 as observed in Bosman et al. (2012). In principle, our results are also compatible with a strong anti-phasic coherence between sender and receiver; however, such a strong coherence has never been observed experimentally, although it would be functionally optimal for selective information routing. This may be because of how the phase-locked states between the sending and receiving populations are established. Previous studies have shown evidence that gamma-activity acts in a feedforward manner, with the upstream populations' gamma-rhythm entraining the gamma in downstream areas (Bosman et al., 2012; Roberts et al., 2013; van Kerkoerle et al., 2014; Bastos et al., 2015; Michalareas et al., 2016; Richter et al., 2017). With this in mind, it is up to the sending population processing the attended stimulus to entrain V4 with its own gamma-rhythm, whereas the population processing the non-attended stimulus fails to entrain V4, remaining primarily uncoupled. Essentially, the presynaptic populations compete to entrain their postsynaptic targets. Although the effect of attention on rates in V1 has been reported to be relatively weak in numerous studies (Moran and Desimone, 1985; Motter, 1993; Luck et al., 1997; McAdams and Maunsell, 1999; Mehta et al., 2000; Salinas and Sejnowski, 2000), modeling studies suggest that even a moderate advantage for the attended signal can be sufficient to entrain the receiving V4 population (Harnack et al., 2015). It has also been reported that the gamma-peak frequency increases slightly with attention in V1 (Bosman et al., 2012), which further promotes its ability to entrain the receiving population (Cannon et al., 2014). 
Our predictions for how SIC is encoded within V4's neural activity relied on the assumption that the information from the sending populations arrives in pulsed packages at V4. In its simplest form, such an encoding can be realized by synergistically combining synchronous dynamics with a rate coding scheme (Ainsworth et al., 2012). Womelsdorf et al., 2012 demonstrated such a scheme in V1, showing that the firing-rate at the peaks of gamma-oscillatory activity is more informative for stimulus orientation. A complementary scheme for encoding information relative to gamma-activity is phase coding. Using the same orientation-selectivity data set, Vinck et al. (2010) showed support for such a phase-coding scheme with the observation that stronger activation by a stimulus leads to spikes emitted earlier in the gamma-cycle. Cumulatively, regardless of precisely how stimulus information is encoded within each gammacycle of the sending populations' neural activity, the crucial component of RBS is that the gamma-rhythmic coordination of said activity increases its postsynaptic impact in a periodic manner, effectively delivering information to the receiving population in the form of gamma-rhythmic packages (Steinmetz et al., 2000; Fries et al., 2001; Azouz and Gray, 2003; Taylor et al., 2005; Zandvakili and Kohn, 2015).

In our results, the higher level of attended information content at excitability peaks in the receiver population in V4 cannot be explained solely by its oscillatory activity. Indeed, having more spikes in the vicinity of V4 excitability peaks has the potential to encode more information at these phases, regardless of any interareal phase coherence between the sending and the receiving population. However, if spikes from sending populations arrived unaligned to V4's gamma-oscillation, we would expect a similar level of SIC phase-modulation for both the attended and the nonattended signals. Further, attention-dependent differences of mean rate in the sender populations do not suggest the big difference observed between the phase-dependent modulation of SIC of attended and of non-attended stimuli, because the mean firing rates in the sending populations are not strongly modulated by attention (Moran and Desimone, 1985; Motter, 1993; Luck et al., 1997; McAdams and Maunsell, 1999; Mehta et al., 2000; Salinas and Sejnowski, 2000). Therefore, we expect that selective phase coherence ensures that information packages for predominantly the attended signal arrive close to the optimal phase at V4 explaining the qualitative difference of the phase modulation between attended and non-attended stimuli.

While selective routing of signals and information based on the synchronization in the gamma-band is a consistent mechanism (Fries, 2005, 2015; Kreiter, 2006, 2020), it is conceptually difficult to draw valid conclusions on information transmission from measuring gamma-coherence within or between cortical areas alone (Buzsáki and Schomburg, 2015). Developing complementary approaches to better link the observed dynamics of neuronal activity to the proposed function in information processing is thus necessary. A significant advance was made by showing that the power of gamma-band activities at two recording sites maximally correlate when these gamma-oscillations are in a favorable phase relationship (Womelsdorf et al., 2007). Consistent with this finding, it was demonstrated that Granger causal influence from upstream to downstream visual areas in the gamma-band is enhanced by attention (Bastos et al., 2015), going along with increased gamma-phase synchronization. However, since gammaactivity is an internal rhythm, the relation to the transmission of stimulus information remained unclear. In our approach, as opposed to investigating internal gamma-rhythms alone, we directly estimate the stimulus information that is contained within
V4's lower frequency $(5-15 \mathrm{~Hz})$ activity by computing the spectral correlation between the luminance flicker of the visual stimuli with V4's neural activity. In consequence, the results establish a causal link between V4 gamma-dynamics and stimulus content, demonstrating a qualitative difference between how attended and non-attended signals are conveyed through V4.

An impact of the pulsed information transmission scheme of RBS on behavior might occur if the animals need to respond quickly to sudden stimulus changes, which result in neural responses with fast initial transients lasting only a few gammacycles (Traschütz et al., 2015). If the relevant information for detecting such changes is predominantly contained in these rapid neural responses, it will be crucial whether it arrives at a favorable or unfavorable phase. Arriving at an unfavorable phase would naturally lead to a larger neural response latency, which could delay successful change detection. Indeed, it has been found that larger response latencies are strongly correlated with longer reaction times, possibly caused by such an effect (Galashan et al., 2013). Further evidence was given by $\mathrm{Ni}$ et al. (2016), who demonstrated that both neural responses and reaction times were modulated by the gamma-phase in V4 at which a sudden stimulus change occurred and also depended on the V1-V4 interareal coherence (Rohenkohl et al., 2018).

Taken together, our findings directly demonstrate that signals carrying information of attended stimuli occur in short packages, tightly locked to the phase of the gamma-band oscillation, in the vicinity of the excitation maximum of the local target population. The results strongly support previous evidence for differential phase synchronization as a mechanism for attention-dependent selective signal routing. In particular, we established the methods to infer and quantify the properties of pulsed transfer schemes in neural data. Since evidence in support for CTC and RBS have also been reported across other visual areas (Womelsdorf et al., 2007; Jia et al., 2013; Besserve et al., 2015) and different brain regions (Buschman and Miller, 2007; Cardin et al., 2009; Siegle et al., 2014), our techniques will allow future studies to pinpoint similar processes in other areas, and to investigate whether the dynamical features exhibited by our data point toward a general principle for flexible information processing throughout the brain.

\section{References}

Ainsworth M, Lee S, Cunningham MO, Traub RD, Kopell NJ, Whittington MA (2012) Rates and rhythms: a synergistic view of frequency and temporal coding in neuronal networks. Neuron 75:572-583.

Atallah BV, Scanziani M (2009) Instantaneous modulation of gamma oscillation frequency by balancing excitation with inhibition. Neuron 62:566-577.

Azouz R, Gray CM (2003) Adaptive coincidence detection and dynamic gain control in visual cortical neurons in vivo. Neuron 37:513-523.

Bastos AM, Vezoli J, Bosman CA, Schoffelen J-M, Oostenveld R, Dowdall JR, De Weerd P, Kennedy H, Fries P (2015) Visual areas exert feedforward and feedback influences through distinct frequency channels. Neuron 85:390-401.

Bédard C, Destexhe A (2012) Modeling local field potentials and their interaction with the extracellular medium. In: Handbook of neural activity measurement, pp 136-191. Cambridge: Cambridge University Press.

Bédard C, Kröger H, Destexhe A (2004) Modeling extracellular field potentials and the frequency-filtering properties of extracellular space. Biophys J 86:1829-1842.

Besserve M, Lowe SC, Logothetis NK, Schölkopf B, Panzeri S (2015) Shifts of gamma phase across primary visual cortical sites reflect dynamic stimulus-modulated information transfer. PLoS Biol 13:e1002257.

Bosman CA, Womelsdorf T, Desimone R, Fries P (2009) A microsaccadic rhythm modulates gamma-band synchronization and behavior. J Neurosci 29:9471-9480.

Bosman CA, Schoffelen J-M, Brunet N, Oostenveld R, Bastos AM, Womelsdorf T, Rubehn B, Stieglitz T, De Weerd P, Fries P (2012) 
Attentional stimulus selection through selective synchronization between monkey visual areas. Neuron 75:875-888.

Buschman TJ, Miller EK (2007) Top-down versus bottom-up control of attention in the prefrontal and posterior parietal cortices. Science 315:1860-1862.

Buzsáki G, Wang XJ (2012) Mechanisms of gamma oscillations. Annu Rev Neurosci 35:203-225.

Buzsáki G, Schomburg EW (2015) What does gamma coherence tell us about inter-regional neural communication? Nat Neurosci 18:484-489.

Cannon J, McCarthy MM, Lee S, Lee J, Börgers C, Whittington MA, Kopell N (2014) Neurosystems: brain rhythms and cognitive processing. Eur J Neurosci 39:705-719.

Cardin JA, Carlén M, Meletis K, Knoblich U, Zhang F, Deisseroth K, Tsai LH, Moore CI (2009) Driving fast-spiking cells induces gamma rhythm and controls sensory responses. Nature 459:663-667.

Delorme A, Mullen T, Kothe C, Akalin Acar Z, Bigdely-Shamlo N, Vankov A, Makeig S (2011) EEGLAB, SIFT, NFT, BCILAB, and ERICA: new tools for advanced EEG processing. Comput Intell Neurosci 2011:130714.

Drebitz E, Haag M, Grothe I, Mandon S, Kreiter AK (2018) Attention configures synchronization within local neuronal networks for processing of the behaviorally relevant stimulus. Front Neural Circuits 12:71.

Drebitz E, Schledde B, Kreiter AK, Wegener D (2019) Optimizing the yield of multi-unit activity by including the entire spiking activity. Front Neurosci 13:83.

Fries P (2005) A mechanism for cognitive dynamics: neuronal communication through neuronal coherence. Trends Cogn Sci 9:474-480.

Fries P (2015) Rhythms for cognition: communication through coherence. Neuron 88:220-235

Fries P, Reynolds JH, Rorie AE, Desimone R (2001) Modulation of oscillatory neuronal synchronization by selective visual attention. Science 291:15601563.

Gabriel S, Lau R, Gabriel C (1996) The dielectric properties of biological tissues: III. Parametric models for the dielectric spectrum of tissues. Phys Med Biol 41:2271-2293.

Galashan FO, Saßen HC, Kreiter AK, Wegener D (2013) Monkey area MT latencies to speed changes depend on attention and correlate with behavioral reaction times. Neuron 78:740-750.

Givre S, Schroeder C, Arezzo JC (1994) Contribution of extrastriate area V4 to the surface-recorded flash VEP in the awake macaque. Vision Res 34:415-428.

Grothe I, Neitzel SD, Mandon S, Kreiter AK (2012) Switching neuronal inputs by differential modulations of gamma-band phase-coherence. J Neurosci 32:16172-16180.

Grothe I, Rotermund D, Neitzel SD, Mandon S, Ernst UA, Kreiter AK, Pawelzik KR (2018) Attention selectively gates afferent signal transmission to area V4. J Neurosci 38:3441-3452.

Harnack D, Ernst UA, Pawelzik KR (2015) A model for attentional information routing through coherence predicts biased competition and multistable perception. J Neurophysiol 114:1593-1605.

Jia X, Tanabe S, Kohn A (2013) $\gamma$ and the coordination of spiking activity in early visual cortex. Neuron 77:762-774.

Kreiter AK (2006) How do we model attention-dependent signal routing? Neural Netw 19:1443-1444

Kreiter AK (2020) Synchrony, flexible network configuration, and linking neural events to behavior. Curr Opin Physiol 16:98-108.

Lavie N (1995) Perceptual load as a necessary condition for selective attention. J Exp Psychol Hum Percept Perform 21:451-468.

Leopold DA, Logothetis NK (1998) Microsaccades differentially modulate neural activity in the striate and extrastriate visual cortex. Exp Brain Res 123:341-345.

Luck SJ, Chelazzi L, Hillyard SA, Desimone R (1997) Neural mechanisms of spatial selective attention in areas V1, V2, and V4 of macaque visual cortex. J Neurophysiol 77:24-42.

Maris E, Oostenveld R (2007) Nonparametric statistical testing of EEG- and MEG-data. J Neurosci Methods 164:177-190.

Maris E, Schoffelen JM, Fries P (2007) Nonparametric statistical testing of coherence differences. J Neurosci Methods 163:161-175.

McAdams CJ, Maunsell JH (1999) Effects of attention on orientation-tuning functions of single neurons in macaque cortical area V4. J Neurosci 19:431-441.
McLelland D, VanRullen R (2016) Theta-gamma coding meets communication-through-coherence: neuronal oscillatory multiplexing theories reconciled. PLoS Comput Biol 12:e1005162.

Mehta SD, Ulbert I, Schroeder CE (2000) Intermodal selective attention in monkeys. I: distribution and timing of effects across visual areas. Cereb Cortex 10:343-358.

Michalareas G, Vezoli J, van Pelt S, Schoffelen J-M, Kennedy H, Fries P (2016) Alpha-beta and gamma rhythms subserve feedback and feedforward influences among human visual cortical areas. Neuron 89:384-397.

Moran J, Desimone R (1985) Selective attention gates visual processing in the extrastriate cortex. Science 229:782-784.

Motter BC (1993) Focal attention produces spatially selective processing in visual cortical areas V1, V2, and V4 in the presence of competing stimuli. J Neurophysiol 70:909-919.

Nandy AS, Nassi JJ, Reynolds JH (2017) Laminar organization of attentional modulation in macaque visual area V4. Neuron 93:235-246.

Nelson MJ, Pouget P, Nilsen EA, Patten CD, Schall JD (2008) Review of signal distortion through metal microelectrode recording circuits and filters. J Neurosci Methods 169:141-157.

Ni J, Wunderle T, Lewis CM, Desimone R, Diester I, Fries P (2016) Gammarhythmic gain modulation. Neuron 92:240-251.

Palmigiano A, Geisel T, Wolf F, Battaglia D (2017) Flexible information routing by transient synchrony. Nat Neurosci 20:1014-1022.

Reynolds JH, Chelazzi L, Desimone R (1999) Competitive mechanisms subserve attention in macaque areas V2 and V4. J Neurosci 19:1736-1753.

Richter CG, Thompson WH, Bosman CA, Fries P (2017) Top-down beta enhances bottom-up gamma. J Neurosci 37:6698-6711.

Roberts MJ, Lowet E, Brunet NM, Ter Wal M, Tiesinga P, Fries P, De Weerd P (2013) Robust gamma coherence between macaque V1 and V2 by dynamic frequency matching. Neuron 78:523-536.

Rohenkohl G, Bosman CA, Fries P (2018) Gamma synchronization between V1 and V4 improves behavioral performance. Neuron 100:953-963.e3.

Salinas E, Sejnowski TJ (2000) Impact of correlated synaptic input on output firing rate and variability in simple neuronal models. J Neurosci 20:61936209.

Salkoff DB, Zagha E, Yüzgeç Ö, McCormick DA (2015) Synaptic mechanisms of tight spike synchrony at gamma frequency in cerebral cortex. J Neurosci 35:10236-10251.

Siegle JH, Pritchett DL, Moore CI (2014) Gamma-range synchronization of fast-spiking interneurons can enhance detection of tactile stimuli. Nat Neurosci 17:1371-1379.

Spyropoulos G, Bosman CA, Fries P (2018) A theta rhythm in macaque visual cortex and its attentional modulation. Proc Natl Acad Sci USA 115: E5614-E5623.

Steinmetz PN, Roy A, Fitzgerald PJ, Hsiao SS, Johnson KO, Niebur E (2000) Attention modulates synchronized neuronal firing in primate somatosensory cortex. Nature 404:187-190.

Taylor K, Mandon S, Freiwald WA, Kreiter AK (2005) Coherent oscillatory activity in monkey area $\mathrm{V} 4$ predicts successful allocation of attention. Cereb Cortex 15:1424-1437.

Traschütz A, Kreiter AK, Wegener D (2015) Transient activity in monkey area MT represents speed changes and is correlated with human behavioral performance. J Neurophysiol 113:890-903.

van Kerkoerle T, Self MW, Dagnino B, Gariel-Mathis M-A, Poort J, van der Togt C, Roelfsema PR (2014) Alpha and gamma oscillations characterize feedback and feedforward processing in monkey visual cortex. Proc Natl Acad Sci USA 111:14332-14341.

Vinck M, Lima B, Womelsdorf T, Oostenveld R, Singer W, Neuenschwander S, Fries P (2010) Gamma-phase shifting in awake monkey visual cortex. J Neurosci 30:1250-1257.

Vinck M, Womelsdorf T, Buffalo EA, Desimone R, Fries P (2013) Attentional modulation of cell-class-specific gamma-band synchronization in awake monkey area V4. Neuron 80:1077-1089.

Womelsdorf T, Schoffelen JM, Oostenveld R, Singer W, Desimone R, Engel AK, Fries $P$ (2007) Modulation of neuronal interactions through neuronal synchronization. Science 316:1609-1612.

Womelsdorf T, Lima B, Vinck M, Oostenveld R, Singer W, Neuenschwander S, Fries P (2012) Orientation selectivity and noise correlation in awake monkey area V1 are modulated by the gamma cycle. Proc Natl Acad Sci USA 109:4302-4307.

Zandvakili A, Kohn A (2015) Coordinated neuronal activity enhances corticocortical communication. Neuron 87:827-839. 